TWO-DIMENSIONAL OFFSETS AND MEDIAL AXIS TRANSFORM

By

Hyeong In Choi, Sung Woo Choi

Chang Yong Han, Tae-wan Kim

Song-Hwa Kwon, Hwan Pyo Moon, Kyeong Hah Roh

and

Nam-Sook Wee

IMA Preprint Series \# 2128

( July 2006)

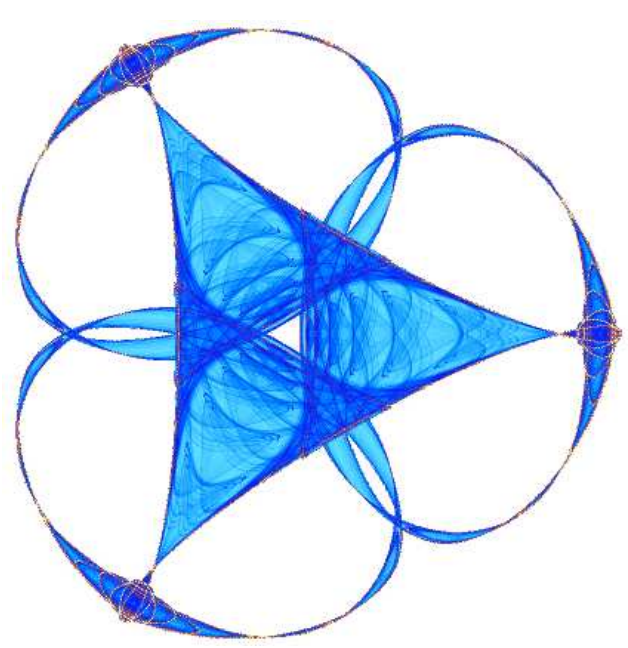

INSTITUTE FOR MATHEMATICS AND ITS APPLICATIONS

UNIVERSITY OF MINNESOTA

400 Lind Hall

207 Church Street S.E.

Minneapolis, Minnesota 55455-0436

Phone: 612-624-6066 Fax: 612-626-7370

URL: http://www.ima.umn.edu 


\title{
Two-dimensional Offsets and Medial Axis Transform
}

\author{
Hyeong In Choi* \\ Department of Mathematics,
}

Seoul National University, Seoul 151-747, Korea

Sung Woo Choi ${ }^{\dagger}$

Department of Mathematics,

Duksung Women's University, Seoul 132-714, Korea

Chang Yong Han ${ }^{\ddagger}$

School of Electronics and Information,

Kyung Hee University, Gyeonggi-do 446-701, Korea

Tae-wan $\mathrm{Kim}^{\S}$

Department of Naval Architecture and Ocean Engineering,

Seoul National University, Seoul 151-744, Korea

Song-Hwa Kwon

Institute of Mathematics and its Application,

University of Minnesota, Minneapolis, MN 55455, USA

Hwan Pyo Moon

Research Lab., Amisys Corporation, Seoul 135-080, Korea

Kyeong Hah Roh

Department of Mathematics and Statistics, Arizona State University, Tempe, AZ 85287, USA

Nam-Sook Wee

Division of Industrial and Mechanical Systems Engineering,

Hansung University, Seoul 136-792, Korea 


\begin{abstract}
We present a mathematical theory of the two-dimensional offset curves from the viewpoint of medial axis transform. We explore the local geometry of the offset curve in relation with the medial axis transform, culminating in the classification of points on the offset curve. We then study the domain decomposition from the viewpoint of offsets, and in particular introduce the concept of monotonic fundamental domain as a device for detecting the correct topology of offsets as well as for stable numerical computation. The monotonic fundamental domains are joined by peaks or valleys of the medial axis transform, or by what we call the critical horizonal section whose algebro-geometric properties are rigorously treated as well.
\end{abstract}

Keywords: Offset curves, Medial axis transform, Domain decomposition, Computer Aided Geometric Design

AMS(MOS) classification: 51N25 (Analytic geometry with other transformation groups)

\title{
1 Introduction
}

Mathematically, an offset curve is a curve of fixed distance away from a given curve. This simple enough definition begets very complicated problems which can be crudely classified into of two types: One is the curve representation problem of the so-called untrimmed offsets, and the other is a more global one, namely, the trimming process of the untrimmed offset curves.

It is easy to see that even if the curve is a polynomial curve, the offset curve need not be a rational curve (of the original parameter). This irrationality may cause some serious problems in the handling of the offset curves in computer aided geometric design. The first kind, i.e., the representation problem, thus received a lot of attention, as far as we know, since Klass [26] had approximated offset curves of cubic splines by using another cubic splines. Since then, there has been a huge amount of work on the approximation of offset curves [7, 8, 9, 10, 15, 22, 23, 27, 28, 30, 33, 34, 36, 37, 38, 41, 42, 43.

In addition to these efforts to approximate offset curves with existing spline curves, other attempts have been made to invent new types of spline curves whose offsets are easy to handle. Meek and Walton 31] studied the offsets of curves consisting of clothoidal splines. In this regard, an important pioneering work has been done by Farouki and Sakkalis [14. They introduced the Pythagorean hodographs, which are special kinds of splines admitting rational offset curves. This feature makes it easier to manipulate the offset curves.

*First author holds joint appointment in the Research Institute of Mathematics, Seoul National University.

†Sung Woo Choi is supported by MOST/KOSEF(Grant \#R01-2005-000-10120-0).

¥This work was supported by the Brain Korea 21 Project in 2005.

$\S$ This work was in part supported by grant No. R01-2005-000-11257-0 from Basic Research Program of Korea Science and Engineering.

ฯ This research was financially supported by Hansung University in the year of 2006. 
Later, Pottmann [39, 40] introduced a rational generalization. Namely, he characterized the rational curves with rational offsets. Jüttler 25] devotes to cubic Pythagorean hodograph $(\mathrm{PH})$ curves which enjoy a number of remarkable properties, such as polynomial arc-length function and existence of associated rational frames. Moreover, geodesic offsets and general offsets are active research areas related to offsets. In 1, general offset curves are treated in the context of Minkowski geometry of the two-dimensional plane, stemming from the consideration of a strictly convex, centrally symmetric given curve as its unit circle. An algorithm to offset curves on offset is presented with tessellated representation for both curves and surfaces by Holla [19].

The second kind, the trimming problem, is of somewhat different nature. This kind of problem occurs due to the presence of a cut or focal loci. An obvious approach to this type of problem would be to compute the intersection of the untrimmed offset curves and decide which portion should be removed to generate the genuine offset curve [21, 42, 31, 30, 16, 3, 32, 24, 20, 29.

A well-known way of getting around this difficulty is to use the medial axis. For domains whose boundary consists of circular arcs and line segments, the Voronoi diagram, which is almost equivalent to the medial axis, has been widely used for a long time in the NC machining industry [6, 17, 18, 35]. The trouble, however, is that most algorithms for finding the medial axis are restricted to the domain whose boundary is made up of circular arcs and line segments. And when the boundary of the domain is free-form, finding the medial axis becomes an even more difficult problem, thereby begging the question.

Some studies on medial axis transform for free-form boundary curves have been done more carefully by few of the present authors: In [4] and [5, a new mathematical theory and algorithm for approximately finding the medial axis transform are presented. They are based on the so-called Domain Decomposition Lemma, which enables one to decompose a complicated domain into simpler, hence easier to handle, subdomains. Chiang et al. 2], have also carried out some works on this feature.

The purpose of this paper is to present a mathematically rigorous theory on offsets from the viewpoint of medial axis transform. We first study the relation between the medial axis transform and offset curves. In particular, we study the local geometry of the medial axis transform and then present our solution of the problem of complete local geometric classification of the points on the offsets. We then present a new approach to the domain decomposition from the viewpoint of offsets, and in particular introduce the concept of monotonic fundamental domain as a device for facilitating the offset computation while preserving the correct topological properties of offsets as well as for stable numerical computation. The monotonic fundamental domains are joined by peaks or valleys of the medial axis transform, or by what we call the critical horizonal section. We also present a theoretical algorithmic procedure of determining whether a given fundamental domain is a critical horizontal section, which is perhaps the most subtle part of the offset computation.

What we are aiming at in the paper is not finding a numerically stable workable algorithm that embodies all the local and global geometric information 
presented in this paper. Rather, our aim is to provide a theoretical foundation or framework for doing so, and for that purpose various results of theoretical bent presented in this paper - the domina decomposition lemma for offsets, the local geometric classification results, the results on the monotonic fundamental domain, and the method of determining the critical horizontal section, and etc. - should prove essential.

\section{Local geometry of medial axis transform from the viewpoint of offset curves}

The offset curve considered throughout our discussion is an inner offset curve of a planar region. We investigate the offset curve by analyzing the medial axis transform of the region. Regarding to the medial axis transform, we will just adopt all the concepts, notations, and results from [4]. Also, as in [4], by the term domain, we mean a noncircular region $\Omega$ which is the closure of a connected bounded open subset in $\mathbb{R}^{2}$ bounded by a finite number of mutually disjoint simple closed curves such that each simple closed curve in $\partial \Omega$ consists of a finite number of pieces of real analytic curves. Even though all the theoretic results in this paper are valid for the region of which boundaries consist of any real analytic curves, some algorithms are valid only for rational curves. Thus, we restrict the domains to those regions of which boundaries only consist of rational curves.

Now, we present here the basic definitions of the offset of a domain and the fundamental relationship between the offset and the medial axis transform.

For a positive number $d$, we define the $d$-offset curve $\mathcal{O}_{d}(\Omega)$ as the inner offset curve to the boundary $\partial \Omega$ at a distance $d$, i.e., the set

$$
\mathcal{O}_{d}(\Omega)=\{\mathbf{p} \in \Omega \mid \operatorname{dist}(\mathbf{p}, \partial \Omega)=d\},
$$

where $\operatorname{dist}(A, B)$ is the Euclidean distance between two sets $A$ and $B$. We also define the $d$-offset region $\Omega_{d}$ as the set inside the $d$-offset curve, i.e.,

$$
\Omega_{d}=\{\mathbf{p} \in \Omega \mid \operatorname{dist}(\mathbf{p}, \partial \Omega) \geq d\} .
$$

A set in $\mathbb{R}^{2}$ is called an offset region, if it is a $d$-offset region of a domain for some $d>0$. Finally, for a subset $A$ of $\mathbb{R}^{2} \times \overline{\mathbb{R}_{+}}$, we denote by $\mathcal{R}(A)$ the region recovered by the disks of $A$, i.e., the set

$$
\mathcal{R}(A)=\bigcup_{(\mathbf{p}, r) \in A} B_{r}(\mathbf{p})=\left\{\mathbf{q} \in \mathbb{R}^{2} \mid \exists(\mathbf{p}, r) \in A, \text { s.t. }|\overline{\mathbf{p q}}| \leq r\right\} .
$$

Then, it is obvious by the definition of the medial axis transform that

$$
\Omega=\mathcal{R}(\mathbf{M A T}(\Omega))
$$

i.e., the domain is the union of all of its maximally inscribed disks. Recall that $\operatorname{MAT}(\Omega)$ denotes the medial axis transform of $\Omega$ [4]. 
To investigate the relationship between the offset and the medial axis transform, we need to define $d$-cutoff $A_{d}$ of $A \subset \mathbb{R}^{2} \times \overline{\mathbb{R}_{+}}$for $d \geq 0$ as

$$
A_{d}=\left\{(\mathbf{p}, r) \in \mathbb{R}^{2} \times \overline{\mathbb{R}_{+}} \mid(\mathbf{p}, r+d) \in A\right\} .
$$

That is, to get $A_{d}$, we first pull $A$ down in the negative $z$-axis by $d$, then remove what is below the $x y$-plane. Then, the remainder is $A_{d}$. Then, it is obvious to see that $\operatorname{MAT}(\Omega)_{d}=\operatorname{MAT}\left(\Omega_{d}\right)$. From this observation, we can get

$$
\begin{aligned}
\mathcal{O}_{d}(\Omega) & =\partial \Omega_{d} \\
& =\partial \mathcal{R}\left(\mathbf{M A T}\left(\Omega_{d}\right)\right) \\
& =\partial \mathcal{R}\left(\mathbf{M A T}(\Omega)_{d}\right) .
\end{aligned}
$$

That is, the $d$-offset curve is the boundary (or envelope) of the region saturated by the disks corresponding to the $d$-cutoff of the medial axis transform of the original domain.

The various geometric aspects of the medial axis transform is revealed in [4]. Especially, we could characterize the medial axis transform as a geometric graph: A geometric graph is a usual topological graph with a finite number of vertices and edges, where a vertex is a point in $\mathbb{R}^{3}$ and an edge is a real analytic curve with finite length and with limits of tangents at the end points.

As a continuing investigation of the geometry of the medial axis transform, here we explore some local geometric property of the cross sections of the medial axis transform cut by horizontal planes. To classify all the possible configurations of the offset curve around its self-intersection points, it is crucial to understand the geometric nature of the cross section, since the self-intersection points of the $d$-offset curve fall on the cross section by the horizontal plane with height $d$. Before we start, let us fix some terminologies that will be used in the subsequent discussion.

For $c \geq 0$, we call a connected component of the set

$$
\{(\mathbf{p}, r) \in \operatorname{MAT}(\Omega) \mid r=c\}
$$

a c-horizontal section of $\operatorname{MAT}(\Omega)$. Since $\operatorname{MAT}(\Omega)$ is a finite geometric graph embedded in $\mathbb{R}^{3}$ as mentioned earlier, it is easy to see that there is a finite number of $c$-horizontal sections of $\operatorname{MAT}(\Omega)$ for given $c \geq 0$, and each horizontal section is either a point or a graph (i.e., a finite geometric graph which is a subset of $\operatorname{MAT}(\Omega)$ ).

Lemma 1. Let $\Omega$ be a domain and let $c>0$. Let $H$ be a c-horizontal section of $\mathbf{M A T}(\Omega)$. Then $H$ is either a point or a $C^{1}$ curve segment (possibly a closed curve). Moreover, if $H$ is a curve segment (or a closed curve), every point in $H$, with possible exception at the end points, is a 2-prong point of $\mathbf{M A T}(\Omega)$.

Proof. Suppose $H$ is not a point. Suppose $(\mathbf{p}, r) \in H$ is an $n$-prong point of $\operatorname{MAT}(\Omega)$, but not an end point of $H$. Let $T(\mathbf{p})$ be the union of the line segments joining $\mathbf{p}$ and the contact points of $B_{r}(\mathbf{p})$. Then, $B_{r}(\mathbf{p}) \backslash T(\mathbf{p})$ has $n$ connected 
components. We denote them by $U_{1}, \ldots, U_{n}$. In [4], it is proved that the medial axis emanating from $\mathbf{p}$ consists, near $\mathbf{p}$, of exactly $n$ curves $\mathbf{s}_{1}, \ldots, \mathbf{s}_{n}$. We may assume each $\mathbf{s}_{i}$ is contained in $U_{i}$ for $i=1, \ldots, n$. We define $\theta_{i}>0$ to be the angle of $U_{i}$ at $\mathbf{p}$ for $i=1, \ldots, n$. Let $\mathbf{r}_{i}$ be the segment of the medial axis transform emanating from $(\mathbf{p}, r)$ corresponding to $\mathbf{s}_{i}$. Let $\phi_{i}$ be the angle at $(\mathbf{p}, r)$ between $\mathbf{r}_{i}$ and the $x y$-plane. The sign of $\phi_{i}$ is chosen to be positive if $\mathbf{r}_{i}$ lies above the plane $\mathbb{R}^{2} \times\{r\}$ near $(\mathbf{p}, r)$. Then, as shown in [4], we have

$$
\tan \phi_{i}=-\cos \frac{\theta_{i}}{2}
$$

for $i=1, \ldots, n$. Note that $n \geq 2$ and at least two of $\phi_{i}$ 's, say $\phi_{1}$ and $\phi_{2}$, are non-negative, since $(\mathbf{p}, r)$ is not an end point of $H$. If $\phi_{1}>0$, then $\theta_{1}>\pi$, which implies $\theta_{2}<\pi$. This in turn implies $\phi_{2}<0$. Thus, we should have $\phi_{1}=\phi_{2}=0$. But then $\theta_{1}=\theta_{2}=\pi$ by Equation (1), so $n$ must be 2 from the fact that $\sum_{i=1}^{n} \theta_{i} \leq 2 \pi$, and $\theta_{i}>0$ for $1 \leq i \leq n$. So the two contact components of $(\mathbf{p}, r)$ must be isolated contact points. For the $C^{1}$ connectivity near $(\mathbf{p}, r)$, one may refer to Section 8.2 in [4].

Definition 1 (Peak, valley, and slope). Let $\Omega$ be a domain and $H$ is a $c$-horizontal section of $\operatorname{MAT}(\Omega)$ for $c \geq 0$. $H$ is called a peak, if it is locally maximal in $r$, i.e., there exists a neighborhood $N$ of $H$ in $\mathbb{R}^{2} \times \overline{\mathbb{R}_{+}}$such that $r \leq c$ for any $(\mathbf{p}, r)$ in $N \cap \mathbf{M A T}(\Omega)$. Similarly, $H$ is called a valley, if it is locally minimal in $r$. Finally if $H$ is neither a peak nor a valley, it is called a slope.

Remark 1. Note that the results in [4] easily implies that the number of peaks and valleys of $\operatorname{MAT}(\Omega)$ is finite.

Remark 2. Note that a $c$-horizontal section can be a peak and a valley at the same time. This is the case when, for example, $\Omega$ is a stadium defined by

$$
\Omega=\left\{(x, y) \in \mathbb{R}^{2} \mid-a \leq y \leq a,-\sqrt{a^{2}-y^{2}}-a \leq x \leq \sqrt{a^{2}-y^{2}}+a\right\},
$$

and $c=a$, the half of the stadium's width.

Theorem 2 (Shape of valley). Let $\Omega$ be a domain. Suppose $V$ is a valley of $\operatorname{MAT}(\Omega)$ and not a 0 -horizontal section of $\mathbf{M A T}(\Omega)$. Then $V$ has the following properties:

(1) $V$ is either a point or a $C^{1}$ (possibly closed) curve segment. If $V$ is a (closed) curve segment, each point on $V$, which is not an end point of $V$, is a 2-prong point of $\operatorname{MAT}(\Omega)$.

(2) None of the points in $V$ (including the possible end points) is a bifurcation point of $\operatorname{MAT}(\Omega)$.

(3) Suppose $V$ is a curve segment. If an end point $(\mathbf{p}, r)$ of $V$ is a 2-prong point of $\mathbf{M A T}(\Omega)$, then $\mathbf{M A T}(\Omega)$ is a $C^{1}$ curve near $(\mathbf{p}, r)$. 
(4) If $V$ is a connected component of $\mathbf{M A T}(\Omega)$, then it is either a closed curve or a curve segment both of whose end points are 1-prong points of $\operatorname{MAT}(\Omega)$.

Proof. The first property is just Lemma 1 Let $(\mathbf{p}, r)$ be a point in $V$. We will show that $(\mathbf{p}, r)$ cannot be a bifurcation point of $\mathbf{M A T}(\Omega)$. We can assume that $V$ is a curve segment and $(\mathbf{p}, r)$ is an end point of $V$. Suppose $(\mathbf{p}, r)$ is an $n$-prong point of $\mathbf{M A T}(\Omega)$ with $n \geq 1$. For $1 \leq i \leq n$, let $\mathbf{r}_{i}, \theta_{i}$, and $\phi_{i}$ be given as in the proof of Lemma 1. Note that $\phi_{i} \geq 0$ for each $i$, since $V$ is a valley. Thus by Equation (1) and the fact that $\sum_{i=1}^{n} \theta_{i} \leq 2 \pi$, we have $n \leq 2$. The third property immediately follows from the similar argument to the latter part of the proof of Lemma 1 .

Now assume $V$ is a connected component of $\operatorname{MAT}(\Omega)$. Note that if $V$ is a closed curve, then it is a connected component of $\operatorname{MAT}(\Omega)$. Suppose $V$ is not a closed curve. Then by the second property, each of the two end points of $V$ is either a 1-prong point or a 2-prong point of $\operatorname{MAT}(\Omega)$. But if one of the end points of $V$ is a 2-prong point of $\operatorname{MAT}(\Omega)$, then $V$ is not a component of $\operatorname{MAT}(\Omega)$. So both of the two end points of $V$ are 1-prong points of $\operatorname{MAT}(\Omega)$.

Lemma 3. Let $\Omega$ be a domain and let $\mathbf{r}:[0,1] \rightarrow \operatorname{MAT}(\Omega), \mathbf{r}(t)=(\mathbf{p}(t), r(t))$ be a continuous path with no self-intersections. (Here we allow the possibility that $\mathbf{r}(0)=\mathbf{r}(1)$.) Suppose there exist $a$ and $b(0<a \leq b<1)$ such that the function $r(t)$ takes a local minimum on $[a, b]$. That is, $r$ is constant on $[a, b]$ and there exists a neighborhood $N$ of $[a, b]$ in $[0,1]$ such that $r(t)<r(\tau)$ for every $t \in[a, b]$ and $\tau \in N \backslash[a, b]$. Then $\mathbf{r}([a, b])$ is a valley of $\mathbf{M A T}(\Omega)$.

Proof. We may assume $r([a, b])>0$. The assumption says that $\mathbf{r}([a, b])$ is a local minimum in the "path" $\mathbf{r}([0,1])$. It remains to show that $\mathbf{r}([a, b])$ is also a local minimum in the "whole" $\mathbf{M A T}(\Omega)$. To show this property, it remains to prove that there is no bifurcation point on $\mathbf{r}([a, b])$. Let $(\mathbf{p}, r)$ be any $n$-prong point of $\mathbf{M A T}(\Omega)$ on $\mathbf{r}([a, b])$. For $1 \leq i \leq n$, let $\mathbf{r}_{i}:[0, \epsilon] \rightarrow \mathbf{M A T}(\Omega)$ and $\theta_{i}, \phi_{i}$ be given as in the proof of Lemma 1. By the assumption, there are at least two $\phi_{i}$ 's, say $\phi_{1}$ and $\phi_{2}$, greater than 0 . By the similar argument to the proof of Lemma 1, $(\mathbf{p}, r)$ is a 2-prong point.

Theorem 4 (Existence of valley between two peaks). Let $\Omega$ be a domain and $P_{1}$ and $P_{2}$ be two (not necessarily distinct) peaks of $\mathbf{M A T}(\Omega)$. Suppose that $\mathbf{r}:[0,1] \rightarrow \mathbf{M A T}(\Omega), \mathbf{r}(t)=(\mathbf{p}(t), r(t))$ is a continuous, not self-intersecting path (possibly closed if $P_{1}=P_{2}$ ) in $\mathbf{M A T}(\Omega)$ with the following properties:

(1) The path $\mathbf{r}$ connects $P_{1}$ and $P_{2}$, i.e., we have $\mathbf{r}(0) \in P_{1}$ and $\mathbf{r}(1) \in P_{2}$.

(2) There exists a $t_{0} \in[0,1]$ such that $\mathbf{r}\left(t_{0}\right) \notin P_{1} \cup P_{2}$.

Then, there is a valley of $\mathbf{M A T}(\Omega)$ on the path $\mathbf{r}$. 
Proof. In view of Lemma 3, it is sufficient to show that there exist $a$ and $b$ $(0<a \leq b<1)$ such that $r(t)$ takes a local minimum on $[a, b]$ in the sense of the lemma. Suppose not. Then $r(t)$ must be either non-decreasing or nonincreasing. Note that $r(t)$ is not constant since there exists a point on $\mathbf{r}$ which is not in $P_{1} \cup P_{2}$. But if $r(t)$ is non-decreasing (respectively, non-increasing), $P_{1}$ (respectively, $P_{2}$ ) cannot be a peak.

\section{Classification of points on offset curves}

Now we are ready to investigate the local geometry of the offset curves of a domain. For a real analytic curve "segment", the geometric and topological features of its offset curve are already familiar in the CAGD context. When the offset distance $d$ is relatively small, its offset curve is very similar to the original one. As $d$ increases, however, the offset curve exhibits irregular points such as cusps, infinite curvature points, or self-intersections. For more information on the irregularity of offset curves, one is referred to [11, 12.

The domain of our concern is bounded by many rational curve segments. Therefore, the offset curve of the domain is a subset of the union of the offset curve segments to the boundary curve segments. In addition to the irregularity of the offset curve segments, caused by the corresponding boundary segments by itself, now the offset curve segments can intersect themselves (depending on the global geometry of the domain) to introduce another kind of irregularity.

Observing that the irregular points always occur on the medial axis of the domain, we will separately treat points of the offset curve that are on the medial axis and off the medial axis after a brief review of the offset of a curve segment.

\subsection{Untrimmed offset curves}

Definition 2. By the untrimmed $d$-offset curve to a boundary curve segment $\mathbf{r}(t)$ for $d>0$, we mean the curve

$$
\mathbf{r}_{d}(t)=\mathbf{r}(t)+d \mathbf{n}(t)
$$

where $\mathbf{n}(t)$ is the unit normal vector of $\mathbf{r}(t)$ pointing inside the domain. Due to the orientation convention of the boundary, the normal vector $\mathbf{n}(t)$ can be obtained by rotating the vector $\mathbf{r}^{\prime}(t) /\left|\mathbf{r}^{\prime}(t)\right|$ counterclockwise by $\pi / 2$.

The unit tangent vector $\boldsymbol{\tau}_{d}(t)$ and curvature $\kappa_{d}(t)$ of the untrimmed offset curve $\mathbf{r}_{d}(t)$ can be readily computed [12] by

$$
\begin{aligned}
\boldsymbol{\tau}_{d}(t) & =\frac{1-d \kappa(t)}{|1-d \kappa(t)|} \boldsymbol{\tau}(t) \\
\kappa_{d}(t) & =\frac{\kappa(t)}{|1-d \kappa(t)|}
\end{aligned}
$$

where $\boldsymbol{\tau}(t)$ is the unit tangent vector and $\kappa(t)$ is the curvature of $\mathbf{r}(t)$. Note that $\mathbf{r}_{d}(t)$ exhibits singularities in its tangent vector (abrupt change of its direction) and curvature (infinite curvature) where $\kappa(t)=1 / d$, i.e., at the center of 
curvature of $\mathbf{r}(t)$. For more analytic properties of the untrimmed offset curve, one is referred to 12 .

\subsection{Points of offset curve off medial axis}

We first consider points of the offset curve which is not on the medial axis.

Theorem 5. Let $\mathbf{p}$ be a point on $\mathcal{O}_{d}(\Omega)$. If $\mathbf{p}$ is not on $\mathbf{M A}(\Omega)$, the following are true:

(1) There is a unique foot point $\mathbf{q}$ of $\mathbf{p}$ on $\partial \Omega$.

(2) Let $\mathbf{r}(t)$ be a parameterization of $\partial \Omega$ near $\mathbf{q}$ with $\mathbf{r}(0)=\mathbf{q}$. There exists an $\epsilon>0$ such that the untrimmed offset curve $\mathbf{r}_{d}(t)$ for $t \in(-\epsilon, \epsilon)$ is $\mathcal{O}_{d}(\Omega)$ near $\mathbf{p}$.

(3) $\mathbf{p}$ is not a singular point of $\mathbf{r}_{d}(t)$.

Proof. If there is more than one foot point of $\mathbf{p}$ on $\partial \Omega$, we should have $\mathbf{p} \in$ $\mathbf{M A}(\Omega)$. Now we prove the second claim. Let $B_{r}(\mathbf{x})$ be the maximal disk of $\Omega$ tangent to $\partial \Omega$ at $\mathbf{q}$. Clearly, we have $r>d$. Since the mapping from the boundary to the medial axis transform (the medial axis transform map in 4]) is continuous, there exists an $\epsilon>0$ such that the corresponding maximal disk contacting at $\mathbf{r}(t)$ has a larger radius value than $d$ for $t \in(-\epsilon, \epsilon)$. We show that the untrimmed offset curve $\mathbf{r}_{d}(t)$ for $t \in(-\epsilon, \epsilon)$ is the $d$-offset curve of $\Omega$ near $\mathbf{p}$. It suffices to show that for any point $\mathbf{r}_{d}(t)$, we have $\operatorname{dist}\left(\mathbf{r}_{d}(t), \partial \Omega\right)=d$. Draw disks $B_{d}\left(\mathbf{r}_{d}(t)\right)$ and $B_{r^{\prime}}\left(\mathbf{p}^{\prime}\right)$, where $B_{r^{\prime}}\left(\mathbf{p}^{\prime}\right)$ is the maximal disk at $\mathbf{r}(t)$. By our choice of $\epsilon$, we have $r^{\prime}>d$. Since the two disks $B_{d}\left(\mathbf{r}_{d}(t)\right)$ and $B_{r^{\prime}}\left(\mathbf{p}^{\prime}\right)$ are tangent to each other at $\mathbf{r}(t)$, we have $B_{d}\left(\mathbf{r}_{d}(t)\right) \subset B_{r^{\prime}}\left(\mathbf{p}^{\prime}\right)$. Hence there is no other point of $\partial \Omega$ which is closer to $\mathbf{r}_{d}(t)$ than $\mathbf{r}(t)$.

To prove the last claim, it suffices to show $\kappa(0)<1 / d$, where $\kappa(t)$ is the curvature of $\mathbf{r}(t)$. Since we have $r \leq 1 / \kappa(0)$ (the radius of the contact disk cannot be larger than the radius of curvature), we have $d<r \leq 1 / \kappa(0)$.

In summary, around the point which is off the medial axis, the offset curve of the domain is essentially the untrimmed offset curve for some boundary curve segments of the domain, and the tangent vector and the curvature are well defined by Equation (2) and (3).

\subsection{Points of offset curve on medial axis}

Now we study points of the offset curve which are on the medial axis. Let $\mathbf{p}$ be a point on $\mathcal{O}_{d}(\Omega)$ and $\mathbf{M A}(\Omega)$. Note that $\mathbf{p}$ is basically a self-intersection point of the offset curve. Let $H$ be the $d$-horizontal section of $\operatorname{MAT}(\Omega)$ having $(\mathbf{p}, d)$. By Lemma 1, $H$ is either a single point or a $C^{1}$ curve segment. We first consider the case in which $H$ is a single point. 


\begin{tabular}{|c|c|c|c|}
\hline prong & valley & peak & slope \\
\hline \hline 1 & yes & yes & no \\
\hline 2 & yes & yes & yes \\
\hline$n(\geq 3)$ & no & yes & yes \\
\hline
\end{tabular}

Table 1: Single-point horizontal section

\section{Single point horizontal section}

Let $\mathbf{p}$ be a point on $\mathcal{O}_{d}(\Omega)$. Suppose, the single point $(\mathbf{p}, d)$ is a $d$-horizontal section of $\operatorname{MAT}(\Omega)$. We list the possible status of $(\mathbf{p}, d)$ as a medial axis transform point in Table 1. Here, there is no 1-prong slope by definition and 3prong valley is also impossible by Theorem 2 . Other possible cases are depicted in Figure 1 .

Case 1 (1-prong valley). The contact disk $B_{d}(\mathbf{p})$ corresponding to $(\mathbf{p}, d)$ is an inscribed osculating disk. The (only one) contact component $C$ of $B_{d}(\mathbf{p})$ is either a point or an arc, but cannot be the whole circle. Let $C(t)$ be a parameterization of $C$ for $t \in[0, \phi]$, where $\phi$ is the angle over which the $\operatorname{arc} C$ extends. (See Figure 2, Since $(\mathbf{p}, d)$ is a valley, we have $0 \leq \phi \leq \pi$. The case $\phi=0$ occurs when $C$ is a point. Let $\mathbf{a}$ and $\mathbf{b}$ be the boundary curve segments of $\Omega$ connected by $C$. (See Figure2, Assume that $\mathbf{a}$ and $\mathbf{b}$ are parameterized in the intervals $(-\epsilon, 0]$ and $[0, \epsilon)$, respectively, such that $\mathbf{a}(0)=C(0)$ and $\mathbf{b}(0)=C(\phi)$.

Theorem 6. The d-offset curve of $\Omega$ near $\mathbf{p}$ is the union of the untrimmed offset curves $\mathbf{a}_{d}$ and $\mathbf{b}_{d}$.

Proof. Since $(\mathbf{p}, d)$ is a valley point and the mapping from the boundary to the medial axis transform is continuous, we can assume that the contact disk at $\mathbf{a}(t)$ or $\mathbf{b}(t)$ has radius greater than $d$ for $t \neq 0$. Now the proof can be the same as that for Theorem 5 .

Remark 3. Note that $\mathbf{a}_{d}$ or $\mathbf{b}_{d}$ has infinite curvature at $\mathbf{p}$ if the curvature of $\mathbf{a}(t)$ or $\mathbf{b}(t)$ is $1 / d$ at $t=0$. Hence, if $\phi=0$, at least one of $\mathbf{a}_{d}$ and $\mathbf{b}_{d}$ must have an infinite curvature at $\mathbf{p}$ while having a continuous tangent direction. And if $\phi=\pi$, one can easily show that none of $\mathbf{a}_{d}$ and $\mathbf{b}_{d}$ can have infinite curvature at $\mathbf{p}$.

In summary, the $d$-offset curve near $\mathbf{p}$ can be decomposed into two curve segments at $\mathbf{p}$. The interior angle of the offset curve at $\mathbf{p}$ is $\pi-\phi$. If $\phi=0$, in particular, the offset curve has a continuous tangent direction but infinite curvature at $\mathbf{p}$.

Case 2 (1-prong peak). Since $(\mathbf{p}, d)$ is a peak point, we can assume that any contact disk near $\mathbf{p}$ (except $B_{d}(\mathbf{p})$ itself) has a smaller radius value than $d$. This means that any point near $\mathbf{p}$ (except $\mathbf{p}$ itself) cannot have $d$ distance to $\partial \Omega$. Hence $d$-offset curve near $\mathbf{p}$ is just $\mathbf{p}$ itself. 


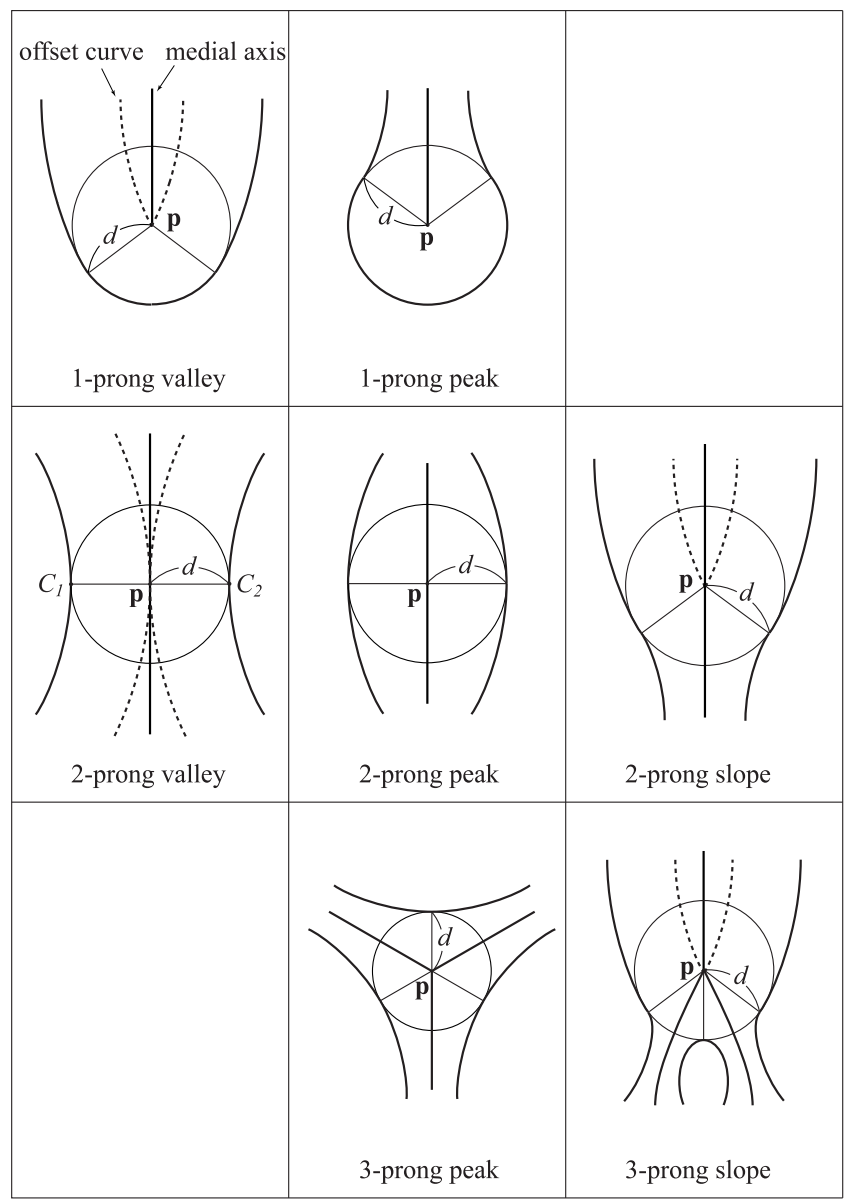

Figure 1: Typical figures near single-point horizontal sections

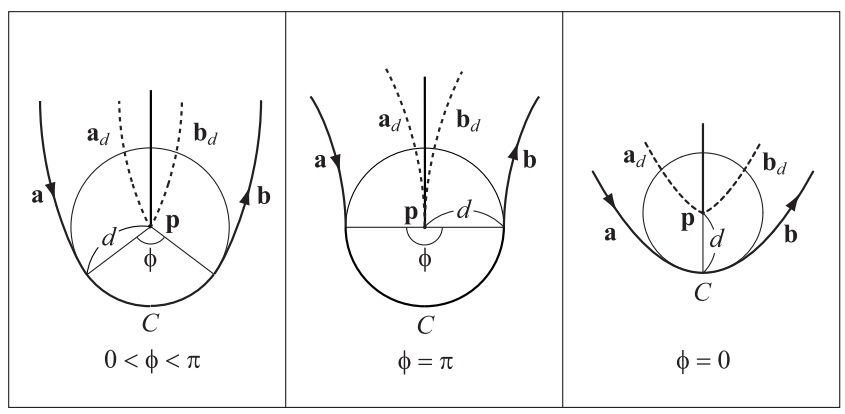

Figure 2: Typical figures near 1-prong valleys 


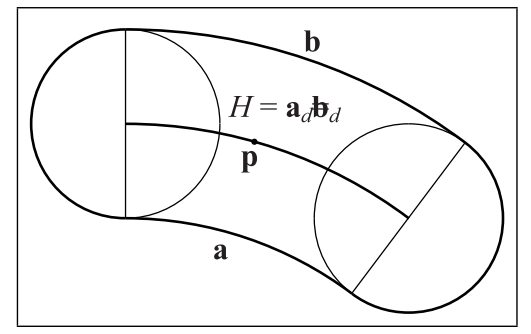

Figure 3: Near a curve segment horizontal section

Case 3 (2-prong valley). If we decompose $\Omega$ at $B_{d}(\mathbf{p})$ into $\Omega_{1}$ and $\Omega_{2}$, then $(\mathbf{p}, d)$ is a single 1 -prong valley point for each $\Omega_{i}$. Now we can apply Theorem 8 Since the contact components $C_{1}$ and $C_{2}$ are isolated points, $\mathcal{O}_{d}(\Omega)$ is the union of $\mathcal{O}_{d}\left(\Omega_{1}\right) \cap A_{1}^{\prime}$ and $\mathcal{O}_{d}\left(\Omega_{2}\right) \cap A_{2}^{\prime}$. Note that we have examined each $\mathcal{O}\left(\Omega_{i}\right)$ in Case 1 with $\phi=\pi$. Hence, the $d$-offset curve near $\mathbf{p}$ is composed of four untrimmed offset curve segments emanating at $\mathbf{p}$ with the same tangent line. None of them have infinite curvature at $\mathbf{p}$. See Figure 1.

Case 4 (2-prong peak). Applying Theorem 8 and Case 2, we can see that the $d$-offset curve near $\mathbf{p}$ is just $\mathbf{p}$ itself.

Case 5 (2-prong slope). Decompose $\Omega$ at $B_{d}(\mathbf{p})$ into $\Omega_{1}$ and $\Omega_{2}$ such that $(\mathbf{p}, d)$ is a valley for $\operatorname{MAT}\left(\Omega_{1}\right)$ and a peak for $\operatorname{MAT}\left(\Omega_{2}\right)$. By Cases 11 and 2 we can see that $\mathcal{O}_{d}(\Omega)$ near $\mathbf{p}$ is just the same as $\mathcal{O}_{d}\left(\Omega_{1}\right)$ near $\mathbf{p}$, which we have described in Case 1 with $\phi \neq 0$.

Case 6 ( $n$-prong peak $(n \geq 3))$. Applying Theorem 8 and Case 2 , we can see that the $d$-offset curve near $\mathbf{p}$ is just $\mathbf{p}$ itself.

Case 7 ( $\boldsymbol{n}$-prong slope $(n \geq 3))$. Decompose $\Omega$ at $B_{d}(\mathbf{p})$ into $\Omega_{1}, \ldots, \Omega_{n}$ where $(\mathbf{p}, d)$ is a valley for only one of the medial axis transforms, say $\mathbf{M A T}\left(\Omega_{1}\right)$ and a peak for $\operatorname{MAT}\left(\Omega_{2}\right), \ldots, \boldsymbol{M A T}\left(\Omega_{n}\right)$. By Cases 1 and 2 , we can see that $\mathcal{O}_{d}(\Omega)$ near $\mathbf{p}$ is just the same as $\mathcal{O}_{d}\left(\Omega_{1}\right)$ near $\mathbf{p}$, which we have described in Case 1] with $\phi \neq 0$.

\section{Curve segment horizontal section}

Now we consider the case in which $H$ is a $C^{1}$ curve segment. First, suppose $(\mathbf{p}, d)$ is not an end point of $H$. Since $H$ is a $d$-horizontal section, for every point $(\mathbf{q}, d)$ of $H$, we have $\operatorname{dist}(\mathbf{q}, \partial \Omega)=d$, i.e., the whole $H$ is a $d$-offset curve of $\Omega$. Since every point of $H$ except the end points is a 2-prong point, there are two corresponding boundary segments near $(\mathbf{p}, d)$. (See Figure 31) And $H$ and the corresponding two boundary curve segments are $d$-offset curves to each other. In summary, the $d$-offset curves of $\Omega$ near $\mathbf{p}$ is a $C^{1}$ untrimmed offset curve to the corresponding boundary curve segments. 


\begin{tabular}{|c|c|c|c|}
\hline prong & "valley" & "peak" & "slope" \\
\hline \hline 1 & - & - & - \\
\hline 2 & yes & yes & - \\
\hline$n(\geq 3)$ & no & yes & - \\
\hline
\end{tabular}

Table 2: At the end point of horizontal section

Case 8 (Curve segment horizontal section). If (p, $d)$ is in the $C^{1}$ curve segment $d$-horizontal section $H$, but it is not an end point of $H$, then the $d$ offset curves of $\Omega$ near $\mathbf{p}$ is a $C^{1}$ untrimmed offset curve to the corresponding boundary curve segments.

Now it remains to investigate whether the case $\mathbf{p}$ is an end point of $H$. We can classify this case as in Table1, Here, we temporarily modify the definitions of the peak, valley, and slope. Note that they were defined for the whole horizontal section. We will call a single point $(\mathbf{p}, r)$ a "peak" of $\boldsymbol{M A T}(\Omega)$, if there is a neighborhood $N$ of $(\mathbf{p}, r)$ such that $r$ is a locally maximal radius value in $N \cap \mathbf{M A T}(\Omega)$. A "valley" and "slope" are similarly modified for the single point case.

We list the possible status of $(\mathbf{p}, d)$, which is an end point of the $C^{1}$ curve segment horizontal section $H$, in Table 2 Note that the concept "slope" is irrelevant here since the radius value of $\mathbf{M A T}(\Omega)$ is constant in the direction of $H$ near $(\mathbf{p}, d)$. And for the 1-prong case, there is no need to artificially divide it into a "valley" and "peak". Typical examples of Table 2 are depicted in Figure 4

Case 9 (1-prong end point). This case is just Case 8 except that there is only one $d$-offset curve segment emanating from $\mathbf{p}$. That is, the $d$-offset curve of $\Omega$ near $\mathbf{p}$ is a $C^{1}$ untrimmed offset curve to the corresponding boundary curve segments emanating from $\mathbf{p}$.

Case 10 (2-prong "valley" end point). By dividing the domain at $B_{d}(\mathbf{p})$, we have two subdomains $\Omega_{1}$ and $\Omega_{2}$ such that $\mathbf{p}$ is a 1-prong valley point of $\operatorname{MAT}\left(\Omega_{1}\right)$ with $\phi=\pi$ (Case 1) and a 1-prong end point of $\operatorname{MAT}\left(\Omega_{2}\right)$ as in Case 9. Hence, the $d$-offset curve of the domain near $\mathbf{p}$ consists of three untrimmed offset curve segments emanating at $\mathbf{p}$ with the same tangent line and none of them have infinite curvature at p. See Figure 4

Case 11 (2-prong "peak" end point). Decompose $\Omega$ at $B_{d}(\mathbf{p})$ into $\Omega_{1}$ and $\Omega_{2}$ such that $\operatorname{MAT}\left(\Omega_{1}\right)=H$. Since $(\mathbf{p}, d)$ is a peak for $\Omega_{2}$, there is no contribution to the $d$-offset curve from the subdomain $\Omega_{2}$ except the point $\mathbf{p}$ itself. Hence the $d$-offset curve near $\mathbf{p}$ is the same as in Case 9 ,

Case 12 ( $n$-prong "peak" end point $(n \geq 3))$. Decompose $\Omega$ at $B_{d}(\mathbf{p})$ into $\Omega_{1}, \ldots, \Omega_{n}$ such that $\operatorname{MAT}\left(\Omega_{1}\right)=H$. Now $(\mathbf{p}, d)$ is a peak for all other $\Omega_{2}, \ldots, \Omega_{n}$. As we have seen in Case 1, there is no contribution to the $d$-offset 


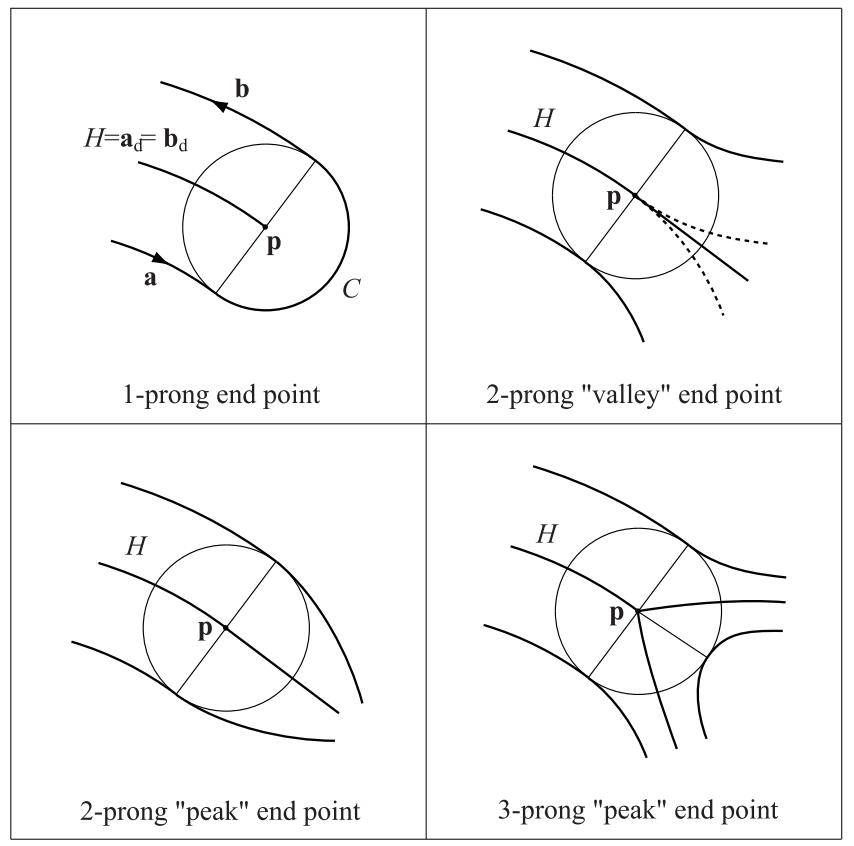

Figure 4: Typical figures near end points of $C^{1}$ curve horizontal sections

curve from the subdomains $\Omega_{2}, \ldots, \Omega_{n}$ except the point $\mathbf{p}$ itself. Hence the $d$-offset curve near $\mathbf{p}$ is the same as in Case 9 .

Now that we have exhausted all the possible cases of the offset curve points of a domain, we can summarize the classification in the following theorem. For the convenience of the enumeration, we introduce the following term.

Definition 3. A point $\mathbf{p}$ of $\mathcal{O}_{d}(\Omega)$ is an $n$-fork point if there are $n$ pieces of curve segments of $\mathcal{O}_{d}(\Omega)$ emanating from $\mathbf{p}$.

Remark 4. We use the term "fork" to distinguish it from the term "prong" used in the description of the medial axis transform in 4, 5.

Theorem 7 (Classification of points on offset curves). Let $\mathbf{p}$ be a point of the d-offset curve of a domain with $d>0$. Then $\mathbf{p}$ is one of the following types; (See Figure 耳)

(0) a 0-fork point, i.e., an isolated point;

(1) a 1-fork point, where a $C^{1}$ curve segment is emanating with finite curvature;

(2) a 2-fork point,

(a) where two $C^{1}$ curve segments are emanating with finite curvature and in opposite tangent directions; 


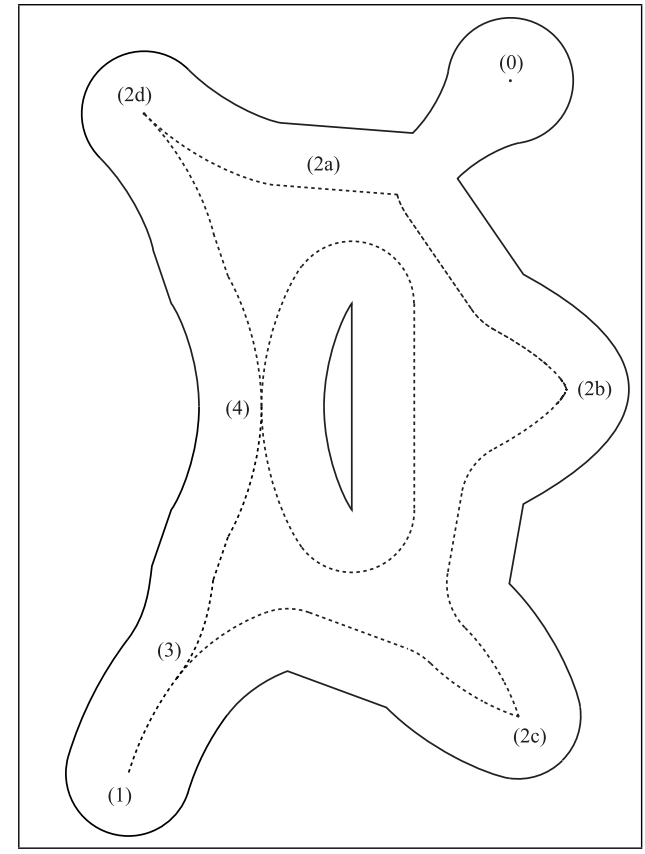

Figure 5: A domain and the offset curve

(b) where two $C^{1}$ curve segments are emanating with opposite tangent directions and at least one of them having an infinite curvature;

(c) where two $C^{1}$ curve segments are emanating with an interior angle $0<\phi<\pi$ and each of them may or may not have an infinite curvature;

(d) where two $C^{1}$ curve segments are emanating with the same tangent direction and finite curvature;

(3) a 3-fork point where three $C^{1}$ curve segments are emanating with finite curvature such that two of them have the same tangent direction but the third has the opposite tangent direction;

(4) a 4-fork point where four $C^{1}$ curve segments are emanating with finite curvature such that two of them have a common tangent direction and the other two have also a common tangent direction but opposite to the first one.

Remark 5. Near a 2-fork point with discontinuous tangent direction, the $d$-offset region is on one side of $d$-offset curve such that the interior angle at that point is less than $\pi$, i.e., there is no "dull" corner point on the boundary of $d$-offset region.

Remark 6. One can show that the number of fork points except (2a) is finite. 
For one curve segment, much literature exists on the geometric and topological features of its offset curve. However, for many pieces of curve segments bounding a planar region, the interference of the offset curve segments with each other makes the analysis much more cumbersome. Using the medial axis transform, which holds the complete data of the region, we were able to rigorously give a full description of the offset curve of the region, albeit people in the CAGD community are well aware by experience. In a subsequent section, we will present an approach to compute the offset curve using the medial axis transform.

\section{Domain decomposition for offsets}

One of the weak points of the medial axis transform is that it is very sensitive to the perturbation of the domain's boundary. A small wrinkle of a smooth segment of the boundary will produce many branches of the medial axis transform coming out toward the wrinkled portion of the boundary. However, the dependence of the medial axis transform on the domain's boundary is in substance local. That is, the wrinkled boundary affects only the contact disks that do contact the boundary. In addition to this localized property, on the other hand, the global information about the medial axis transform can be obtained by combining local information about the medial axis transform (Domain Decomposition Lemma (4).

The domain decomposition method was the basic tool for the construction of the medial axis transform in 5 . Now, we have at hand the relation between the medial axis transform and the offset curve. It is advantageous if we know how the domain decomposition method can be applied to the offset curve construction.

Theorem 8 (Domain decomposition lemma for offset). Let $(\mathbf{p}, r)$ be an $n$-prong point of $\mathbf{M A T}(\Omega)$ and let $C_{1}, \ldots, C_{n}$ be the corresponding contact components. Let $B_{r}(\mathbf{p})$ be the corresponding contact disk. Suppose $A_{1}, \ldots, A_{m}$ are the connected components of $\Omega \backslash B_{r}(\mathbf{p})$. Let $S_{i}=\partial B_{r}(\mathbf{p}) \cap \overline{A_{i}}$, a segment of $\partial B_{r}(\mathbf{p})$. Let $U_{i}$ be the union of the closed sectors of $B_{r}(\mathbf{p})$ corresponding to $S_{i}$. That is, $U_{i}=\left\{\mathbf{z} \in \overline{\mathbf{p q}} \mid \mathbf{q} \in S_{i}\right\}$. Denote $\Omega_{i}=A_{i} \cup B_{r}(\mathbf{p})$ and $A_{i}^{\prime}=A_{i} \cup U_{i}$ for $i=1, \ldots, m$. Then, the $d$-offset curve $\mathcal{O}_{d}(\Omega)$ can be decomposed into the following components;

(1) $\mathcal{O}_{d}\left(\Omega_{i}\right) \cap A_{i}^{\prime}$ for $i=1, \ldots, m$,

(2) $d$-offset curves to the arcs $C_{j}$, for $j=1, \ldots, n$.

Furthermore, each pair of the above components can, at best, share their end points. See Figure 6 .

Proof. Let $T_{j}$ be the sector of $\partial B_{r}(\mathbf{p})$ corresponding to the contact component $C_{j}$, i.e., $T_{j}=\left\{\mathbf{z} \in \overline{\mathbf{p q}} \mid \mathbf{q} \in C_{j}\right\}$. It is easy to see that $\mathcal{O}_{d}(\Omega) \cap T_{j}$ is the $d$-offset curve to the $\operatorname{arc} C_{j}$, which is just the same $\operatorname{arc}$ as $C_{j}$ with the same center but $r-d$ radius. (If $d>r$, no such arc exists.) 


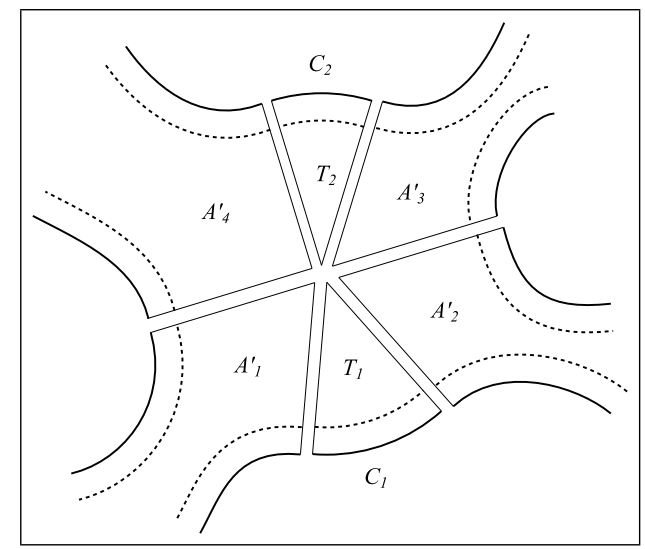

Figure 6: The partition of the offset curve

Since the union of all $A_{i}^{\prime}$ and $T_{j}$ is the whole domain $\Omega$, we have

$$
\mathcal{O}_{d}(\Omega)=\bigcup_{i=1}^{m}\left(\mathcal{O}_{d}(\Omega) \cap A_{i}^{\prime}\right) \cup \bigcup_{j=1}^{n}\left(\mathcal{O}_{d}(\Omega) \cap T_{j}\right) .
$$

Now let us show that $\mathcal{O}_{d}\left(\Omega_{i}\right) \cap A_{i}^{\prime}=\mathcal{O}_{d}(\Omega) \cap A_{i}^{\prime}$ for each $i$. It suffices to show that $\operatorname{dist}\left(\mathbf{z}, \partial \Omega_{i}\right)=\operatorname{dist}(\mathbf{z}, \partial \Omega)$ for any $\mathbf{z} \in A_{i}^{\prime}$.

Denote $\operatorname{dist}\left(\mathbf{z}, \partial \Omega_{i}\right)$ by $d$. Let $\mathbf{w}$ be a foot point of $\mathbf{z}$ on $\partial \Omega_{i}$. We first show that $\mathbf{w}$ is on $\partial \Omega$ and thus we have $\operatorname{dist}\left(\mathbf{z}, \partial \Omega_{i}\right) \geq \operatorname{dist}(\mathbf{z}, \partial \Omega)$. Suppose $\mathbf{w}$ is not on $\partial \Omega$ but on $\tilde{S}_{i}$. Since $\mathbf{z}$ is inside $A_{i}^{\prime}$ (including the boundary) but $\mathbf{w}$ is outside $A_{i}^{\prime}$, $\overline{\mathbf{z W}}$ intersects the boundary of $A_{i}^{\prime}$, which is $\left(\partial A_{i} \cap \partial \Omega\right) \cup \overline{\mathbf{p q _ { 1 }}} \cup \overline{\mathbf{p q}_{2}}$, where $\mathbf{q}_{1}$ and $\mathbf{q}_{2}$ are the end points of $S_{i}$. By the assumption, $\overline{\mathbf{z W}}$ must intersect $\overline{\mathbf{p q}}$ or $\overline{\mathbf{p q}_{2}}$. (Otherwise, the intersection point on $\partial A_{i} \cap \partial \Omega$ realizes $\operatorname{dist}\left(\mathbf{z}, \partial \Omega_{i}\right)$ with a smaller value than $d$.) Then it is easy to see that there are points on $\tilde{S}_{i}$ realizing $\operatorname{dist}\left(\mathbf{z}, \partial \Omega_{i}\right)$ with a smaller value than $d$.

Now we show that $\operatorname{dist}\left(\mathbf{z}, \partial \Omega_{i}\right) \leq \operatorname{dist}(\mathbf{z}, \partial \Omega)$. Let $\mathbf{v}$ be a foot point of $\mathbf{z}$ on $\partial \Omega$ realizing $\operatorname{dist}(\mathbf{z}, \partial \Omega)$. If $\mathbf{v}$ is on $\partial \Omega_{i}$, we have $\operatorname{dist}\left(\mathbf{z}, \partial \Omega_{i}\right) \leq|\overline{\mathbf{z v}}|=\operatorname{dist}(\mathbf{z}, \partial \Omega)$. If $\mathbf{v}$ is not on $\partial \Omega_{i}$, then $\overline{\mathbf{z V}}$ must intersect $\partial \Omega_{i}$. Let $\mathbf{y}$ be the intersection point. Then we have $\operatorname{dist}\left(\mathbf{z}, \partial \Omega_{i}\right) \leq|\overline{\mathbf{z y}}| \leq|\overline{\mathbf{z v}}|=\operatorname{dist}(\mathbf{z}, \partial \Omega)$.

Therefore, we proved $\mathcal{O}_{d}\left(\Omega_{i}\right) \cap A_{i}^{\prime}=\mathcal{O}_{d}(\Omega) \cap A_{i}^{\prime}$. Finally, note that any pair of distinct $A_{i}^{\prime}$ and $A_{k}^{\prime}$ or $A_{i}^{\prime}$ and $T_{j}$ can at best share their boundary line segments.

Although the medial axis transform does not allow in general any closedform expression via the parameterization of the boundary, one can find the contact disk corresponding to any boundary points. In [5] and [13], algorithms to pinpoint the medial axis transform point $(\mathbf{p}, r)$ corresponding to a given boundary point $\mathbf{q}$ are presented. In [5], one draws a suspect disk contacting the boundary curve at $\mathbf{q}$ and intersecting the other boundary curve, and then reduces this disk until it is tangent to the other boundary curve. On the other 


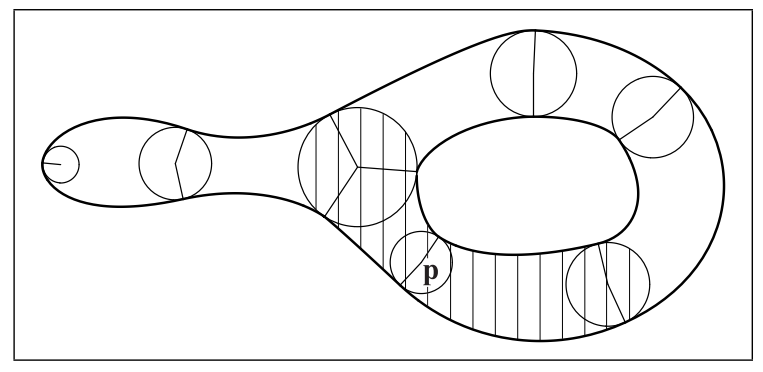

Figure 7: A subdomain around $\mathbf{p}$

hand, the second algorithm [13, called curve/curve-bisector, is based on the observation that $\mathbf{p}$ is the intersection point of the normal line of the boundary curve at $\mathbf{q}$ and the point/curve bisector of $\mathbf{q}$ and the other boundary curve. These algorithms are the 'workhorse' of our algorithm and we will call them, in a general term, the medial axis transform engines.

The medial axis transform can be approximated by interpolating the points found by the medial axis transform engines. The very property of the medial axis transform that makes this process possible is that the medial axis transform is a finite geometric graph.

If we have an approximation of the medial axis transform, we can find the shape of a domain since the medial axis transform is a strong deformation retract of the domain. To get a more accurate approximation of the medial axis transform, we must find more contact disks using the medial axis transform engines, and include them in the interpolation scheme. Now, when we run the medial axis transform engines to find those additional contact disks, we do not have to consider the whole domain. We only need to consider the region around such contact disks. For example, if we want to find a medial axis point $\mathbf{p}$ in Figure 7, we can concentrate on the shaded region only, not the whole domain; this is the idea of the domain decomposition. Thus, each contact disk decomposes the domain into subdomains. Especially, if we find all the bifurcation points (where at least three edges meet) of the medial axis transform, then the original domains are decomposed into subdomains whose medial axis transforms are piecewise real analytic curves. We call such a subdomain a fundamental domain, and Domain Decomposition Lemma 44 says that it is sufficient to deal with fundamental domains only. (For the ease of computation, we can assume that the boundaries of the fundamental domains are rational rather than piecewise-rational after placing a disk at every juncture in the boundary curve.)

The idea of domain decomposition is also the cornerstone of our approach to offset. We have seen in Theorem 8 that once the offset curves of the original boundary curve segments in fundamental domains are constructed, then domain decomposition links them into the whole offset curve of the domain. In Figure 8 for example, the offset curve of each subdomain does not interfere with the offset curves of other subdomains, but can be easily linked with each other, forming the offset curve of the whole domain. 


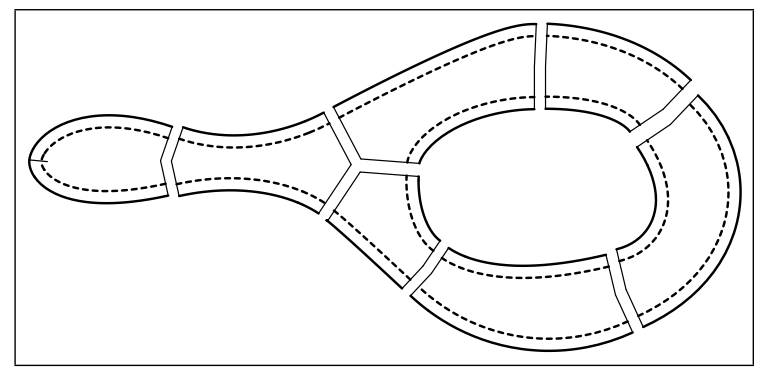

Figure 8: Domain decomposition for offset curves

In addition, the self-intersection points of the $d$-offset curve lie on the medial axis where its radius is $d$. As we have seen in Lemma 1, the set of all medial axis transform points whose radius component is $c$, for a given $c>0$, are composed of a finite number of points or piecewise $C^{1}$ curves. The various geometries of a $c$-horizontal section and the offset curve near self-intersection points are thoroughly discussed in the preceding sections. Hence, $d$-horizontal section must be located to capture the exact nature of a $d$-offset curve.

However, given a fundamental domain $\Omega_{F}$ and $d>0$, locating such $d$ horizontal section in $\Omega_{F}$ is difficult. If we assume that $\operatorname{MAT}\left(\Omega_{F}\right)$ is represented by a piecewise real analytic curve $(\mathbf{p}(t), r(t))$, the problem is equivalent to solving the equation $r(t)=d$. But we do not have any closed form expression for the function $r(t)$. What we have is some engines that compute the value of $\mathbf{p}(t)$ and $r(t)$ for given $t$. To incorporate such engines into a stable root-finding schemes, one must carefully examine the problem space beforehand.

Thus, to prepare, we are going to further decompose the domain such that the medial axis transform of each subdomain has a monotone increasing/decreasing radius component. Once this decomposition is done, the solution of the equation $r(t)=d$ can be efficiently and stably obtained by any traditional numerical methods, such as Newton-Raphson method. Thus, a special $c$-horizontal section, which has a local maximum or minimum radius value, called peak or valley, respectively, needed to be located in advance. To do so, we use iterative procedure to approximately locate peaks and valleys. If we know, in advance, that there are no other points nearby which have extreme value in radius, these iterative algorithms work well. The problem occurs when there are other, sometimes infinite, points nearby which have extreme value in radius. These must be roughly counted by looking at the boundary geometry. In particular, it is advantageous to decompose the domain such that each curvature of the boundary curve segments of its subdomains does not change sign.

In doing so, one can also encounter the situation where there are infinitely many points which have extreme value in radius. Since the medial axis transform our domain is real analytic curve, infinitely many such points occur only when the fundamental domain is parallel; this is, the two boundary curve segments are offset to each other. Using Bezout's theorem, we will provide an algorithm to determine whether a given domain is parallel. 
In Section 5, we will describe the decomposition of the domain into subdomains, whose medial axis transform has a monotone radius components. Then in Section [6] we can easily compute the points satisfying the equation $r(t)=d$.

\section{Critical horizontal section}

We now discuss how to locate horizontal sections with a critical radius value. Such horizontal sections will be shortly called critical horizontal sections. Peaks and valleys are kinds of critical horizontal sections.

We begin with fixing some conventions on the boundary which will be used throughout this discussion. We assume that every curve segment $\mathbf{r}(t)$ composing the boundary is positively-oriented. That is, the parameter $t$ is so chosen that if one is going along the curve in the direction of increasing $t$, then the domain remains to the left.

Let $\Omega_{F}$ be a fundamental domain at hand and $\mathbf{r}$ and $\mathbf{s}$ be its two boundary curves of the domain defined on the interval $[0,1]$. Let $\mathbf{p}(t)$ be the corresponding medial axis point of $\mathbf{r}(t)$, and let $\mathbf{s}(u)$ be the foot point of $\mathbf{p}(t)$ on $\mathbf{s}$. The opening angle $\theta(t)$ of $\mathbf{p}(t)$ (or $\mathbf{r}(t)$ ) is the angle from $\overrightarrow{\mathbf{p}(t) \mathbf{r}(t)}$ to $\overrightarrow{\mathbf{p}(t) \mathbf{s}(u)}$ in the counterclockwise direction. If $\mathbf{p}(t)$ is on a critical horizontal section, then $\theta(t)=\pi$ since we know that

$$
\cos \frac{\theta(t)}{2}=-\tan \phi(t)
$$

where $\phi(t)$ is the angle between the tangent vectors $\left(\mathbf{p}^{\prime}(t), r^{\prime}(t)\right)$ and $\mathbf{p}^{\prime}(t)$. Thus locating critical horizontal sections is equivalent to locating $\pi$-opening angle sections.

We have found that it is advantageous to further decompose the fundamental domain such that the curvature of its boundary curves have constant sign. To do that, we need to locate the boundary parameter where the curvature changes its sign. Candidates are those parameters where the curvature is discontinuous or zero.

The curvature $\kappa(t)$ of a boundary curve $\mathbf{r}(t)$ is defined by

$$
\kappa(t)=\frac{\operatorname{det}\left(\mathbf{r}^{\prime}(t), \mathbf{r}^{\prime \prime}(t)\right)}{\left|\mathbf{r}^{\prime}(t)\right|^{3 / 2}} .
$$

According to this definition, a curve with positive (resp., negative) curvature will bend to the left (resp., right) as one is going along the curve in the direction of increasing $t$. See Figure 9 .

For a rational $\mathbf{r}(t)=(x(t) / w(t), y(t) / w(t))$, we have

$$
\kappa=\frac{w^{3}\left(x\left(y^{\prime} w^{\prime \prime}-y^{\prime \prime} w^{\prime}\right)+y\left(w^{\prime} x^{\prime \prime}-w^{\prime \prime} x^{\prime}\right)+w\left(x^{\prime} y^{\prime \prime}-x^{\prime \prime} y^{\prime}\right)\right)}{\left(\left(x w^{\prime}-x^{\prime} w\right)^{2}+\left(y w^{\prime}-y^{\prime} w\right)^{2}\right)^{3 / 2}}
$$

Thus, if $\mathbf{r}(t)$ is a rational curve of degree $n$, locating zero-curvature points of $\mathbf{r}$ amounts to solving $3 n-4$ order univariate polynomial equations ( $2 n-4$ if polynomial curve). At the zero-curvature points on the boundary, we can get the 


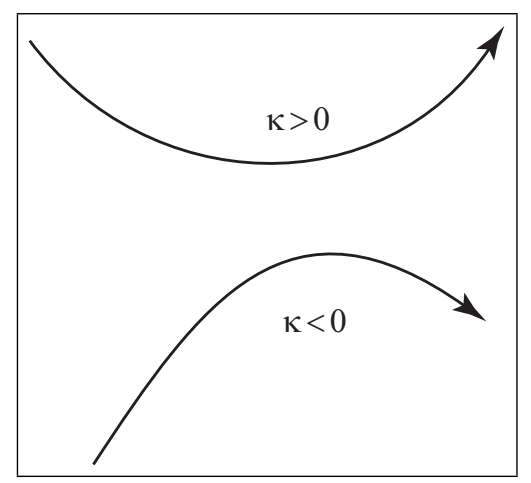

Figure 9: Curvature sign convention

corresponding medial axis transform points. By these medial axis transform points, the original domain is further decomposed into fundamental domains whose boundary curves have constant curvature sign except possibly at the end points. We can classify these fundamental domains into four possible cases according to the curvature sign. For convenience, we adopt the following conventions for the curvatures $\kappa_{\mathbf{r}}$ and $\kappa_{\mathbf{s}}$ of $\mathbf{r}$ and $\mathbf{s}$, respectively: we denote $\kappa_{*}>0$ if $\kappa_{*}(t)>0$ for all $t$ except possibly for $t=0,1$ and $\kappa_{*} \geq 0$ if $\kappa_{*}>0$ or $\kappa_{*}(t) \equiv 0$ for all $t$. Similarly $\kappa_{*}<0$ and $\kappa_{*} \leq 0$ are defined.

Now we describe how to locate $\pi$-opening angle sections case by case.

Case 1: $\kappa_{\mathbf{r}} \leq 0$ and $\kappa_{\mathbf{s}} \leq 0$

For any given contact point $\mathbf{r}(t)$ on $\mathbf{r}$, let $\mathbf{s}(u)$ be the corresponding contact point of $\mathbf{r}(t)$ on $\mathbf{s}$. Note that $u=u(t)$ is a decreasing function of $t$ due to the orientation convention of the boundary curve. Let $\alpha(t)$ and $\beta(u)$ be the angles of $\mathbf{r}^{\prime}(t)$ and $\mathbf{s}^{\prime}(u)$ with respect to the positive $x$-axis, respectively such that

$$
\theta(t)=\beta(u)-\alpha(t),
$$

which means that the opening angle $\theta(t)$ is equal to the angle from $\mathbf{r}^{\prime}(t)$ to $\mathbf{s}^{\prime}(u)$. (See Figure 10,) Since $\beta(u)$ is a decreasing function of $u$ and $u=u(t)$ and $\alpha(t)$ is decreasing functions of $t, \theta(t)$ is an increasing function of $t$.

From this observation, we have the following result.

Proposition 9. If $\theta(0)>\pi$ or $\theta(1)<\pi$, there is no critical horizontal sections in $\Omega_{F}$. If $\theta(0) \leq \pi$ and $\theta(1) \geq \pi$, there exists exactly one critical horizontal section, which is a valley in $\Omega_{F}$. In this case, if both $\mathbf{r}$ and $\mathbf{s}$ are line segments, i.e., $\kappa_{\mathbf{r}} \equiv 0$ and $\kappa_{\mathbf{s}} \equiv 0$, then the whole $\mathbf{M A T}\left(\Omega_{F}\right)$ is a critical horizontal section. Otherwise, the critical horizontal section is a single point.

Case 2: $\kappa_{\mathbf{r}} \geq 0, \kappa_{\mathbf{s}} \geq 0$

This case is analogous to the Case 1. Proposition 9 can be adapted as follows. 


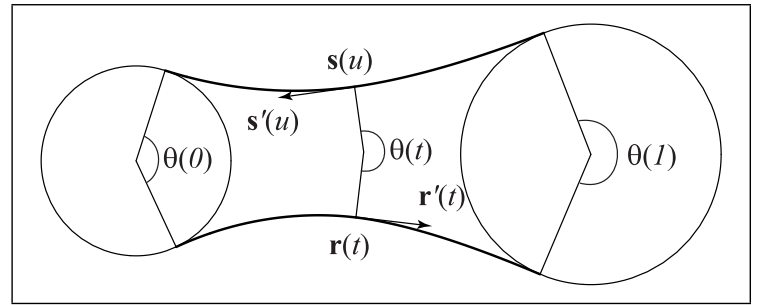

Figure 10: $\kappa_{\mathbf{r}} \leq 0, \kappa_{\mathbf{s}} \leq 0$

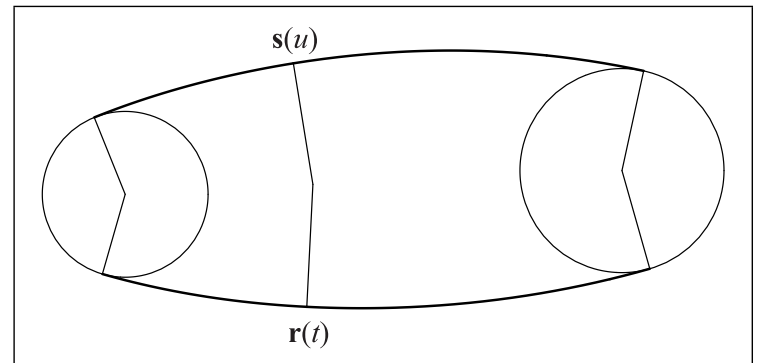

Figure 11: $\kappa_{\mathbf{r}} \geq 0, \kappa_{\mathbf{s}} \geq 0$

Proposition 10. If $\theta(0)<\pi$ or $\theta(1)>\pi$, there is no critical horizontal sections in $\Omega_{F}$. If $\theta(0) \geq \pi$ and $\theta(1) \leq \pi$, there exists exactly one critical horizontal section, which is a peak in $\Omega_{F}$. In this case, if both $\mathbf{r}$ and $\mathbf{s}$ are line segments, i.e., $\kappa_{\mathbf{r}} \equiv 0$ and $\kappa_{\mathbf{s}} \equiv 0$, then the whole $\mathbf{M A T}\left(\Omega_{F}\right)$ is a critical horizontal section. Otherwise, the critical horizontal section is a single point.

The locating algorithm is similar as in Case 1.

Case $3 *$ : $\kappa_{\mathrm{r}}>0, \kappa_{\mathrm{s}}<0$

In this case, we cannot determine the existence of peak or valley, or how many there are, if any, with only the data of $\theta(0)$ and $\theta(1)$. In general, one can formulate an example which has as many peaks and valleys as one wishes with given $\theta(0)$ and $\theta(1)$ and under the condition $\kappa_{\mathbf{r}}>0, \kappa_{\mathbf{s}}<0$. For example, in Figure 12 we have one peak and one valley between $\mathbf{p}_{1}$ and $\mathbf{p}_{3}$ whereas none of them between $\mathbf{p}_{2}$ and $\mathbf{p}_{3}$. (Note that $\mathbf{p}_{1}$ and $\mathbf{p}_{2}$ have the same opening angle and radius.) Moreover, we cannot exclude the possibility that portions of $\mathbf{r}$ and $\mathbf{s}$ are offset curves to each other. We begin with this problem. Since MAT $(\Omega)$ is a real analytic curve at every generic 2-prong point and our fundamental domain consists of generic 2-prong only (except possibly at the end points), $\operatorname{MAT}\left(\Omega_{F}\right)$ is a real analytic curve. By the real analyticity of radius component of $\operatorname{MAT}\left(\Omega_{F}\right)$, we have the following:

${ }^{*}$ By changing the roles of $\mathbf{r}$ and $\mathbf{s}$, we can also cover the case that $\kappa_{\mathbf{r}}<0, \kappa_{\mathbf{s}}>0$. 


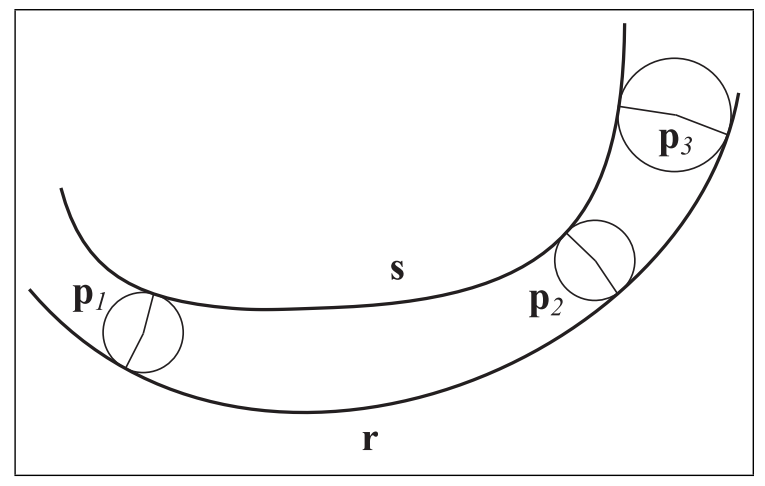

Figure 12: $\kappa_{\mathbf{r}}>0, \kappa_{\mathbf{s}}<0$

Theorem 11. Either the whole $\mathbf{M A T}\left(\Omega_{F}\right)$ is a c-horizontal section for some $c>0$, or every c-horizontal section of $\mathbf{M A T}\left(\Omega_{F}\right)$ consists of a finite number of points.

That is, either the whole $\mathbf{r}$ and $\mathbf{s}$ are offset curves to each other (in this case we call $\Omega_{F}$ a parallel fundamental domain), or there is a finite number of critical radius points. The decision of whether or not $\Omega_{F}$ is parallel can be made in finite steps. The criterion for this is given in Theorem 13. Bezout's theorem is crucial in proving this theorem.

Theorem 12 (Bezout's Theorem). Two relatively prime polynomials $f, g \in$ $\mathbb{C}[x, y]$ of degree $d_{1}$ and $d_{2}$ can have at most $d_{1} d_{2}$ simultaneous solutions.

For a given polynomial $h(x, y)$, the equation $h(x, y)=0$ usually defines a curve in $\mathbb{R}^{2}$. But in some cases, the solution of $h(x, y)=0$ consists of a single point. (For example $h(x, y)=x^{2}+y^{2}$.) We call such a solution an isolated solution. To be precise, a point $\left(x_{0}, y_{0}\right)$ is called an isolated solution of the equation $h(x, y)=0$, if for some neighborhood $V$ of $\left(x_{0}, y_{0}\right)$, there is no other solution than $\left(x_{0}, y_{0}\right)$ in $V$.

The next is then an easy corollary of the Bezout's theorem.

Corollary. Let $h(x, y)$ be an irreducible polynomial over $\mathbb{R}$ of positive degree $d$. Then $h(x, y)=0$ can have at most $d(d-1)$ isolated solutions.

Proof. If $\left(x_{0}, y_{0}\right)$ is an isolated solution of $h(x, y)=0$, then $\frac{\partial h}{\partial x}\left(x_{0}, y_{0}\right)=$ $\frac{\partial h}{\partial y}\left(x_{0}, y_{0}\right)=0$. That is, $\left(x_{0}, y_{0}\right)$ is a simultaneous solution of

$$
\begin{aligned}
h(x, y) & =0 \\
\frac{\partial h}{\partial x}(x, y) & =0 \\
\frac{\partial h}{\partial y}(x, y) & =0 .
\end{aligned}
$$


Since $h$ is irreducible, $h$ and $\frac{\partial h}{\partial x}$ (or $\frac{\partial h}{\partial y}$ ) are relatively prime. Now the result follows from Bezout's theorem.

Theorem 13. Let $\Omega_{F}$ be a fundamental domain with boundary curves $\mathbf{r}$ and $\mathbf{s}$. Suppose $\mathbf{r}$ and $\mathbf{s}$ are rational curves of degree $m$ and $n$, respectively. Then $\Omega_{F}$ is parallel if and only if:

(i) the radii at ends are same, i.e., $r(0)=r(1)$, and

(ii) For some distinct $N+1$ points $0=t_{0}, \ldots, t_{N}=1$, where $N=(2 m+2 n-$ $1)^{2}$, we have $\theta\left(t_{0}\right)=\cdots=\theta\left(t_{N}\right)=\pi$.

If $\Omega_{F}$ is parallel, then for any $N+1$ points, the second condition is satisfied. What this theorem says is that any one set $S$ of $N+1$ points is enough. If one of $t_{i}$ in $S$ does not satisfy the condition, then $\Omega_{F}$ is not parallel. Otherwise, i.e., if every $t_{i}$ in $S$ does satisfy the condition, then $\Omega_{F}$ is parallel.

Proof. One way is obvious. Conversely, let $\mathbf{s}\left(u_{i}\right), i=0, \ldots, N$ be the corresponding contact points of $\mathbf{r}\left(t_{i}\right)$ on $\mathbf{s}$. Since $\theta\left(t_{i}\right)=\pi,\left(t_{i}, u_{i}\right)$ are solutions of

$$
\begin{array}{r}
\mathbf{r}^{\prime}(t) \cdot(\mathbf{r}(t)-\mathbf{s}(u))=0 \\
\mathbf{s}^{\prime}(u) \cdot(\mathbf{s}(u)-\mathbf{r}(t))=0 .
\end{array}
$$

If one substitutes

$$
\begin{aligned}
\mathbf{r}(t) & =\frac{1}{c(t)}(a(t), b(t)) \\
\mathbf{s}(u) & =\frac{1}{f(u)}(d(u), e(u))
\end{aligned}
$$

the above equations are reduced to polynomial equations

$$
\begin{aligned}
& P(t, u)=(a f-c d)\left(a^{\prime} c-a c^{\prime}\right)+(b f-c e)\left(b^{\prime} c-b c^{\prime}\right)=0 \\
& Q(t, u)=(a f-c d)\left(d^{\prime} f-d f^{\prime}\right)+(b f-c e)\left(e^{\prime} f-e f^{\prime}\right)=0 .
\end{aligned}
$$

Note that both $P$ and $Q$ have total degree of, at most, $2 m+2 n-1$. In view of Bezout's theorem, $P$ and $Q$ must have a common factor of positive degree. Let $h$ be the greatest common divisor of $P$ and $Q$. Suppose $h$ is written as a product

$$
h=h_{1} \cdots h_{r}
$$

of irreducibles over $\mathbb{R}$ of positive degree $d_{1}, \ldots, d_{r}$. Now if we write $P=P_{1} h$ and $Q=Q_{1} h$, then $\left(t_{i}, u_{i}\right)$ are simultaneous solutions of

$$
\begin{aligned}
P_{1}(t, u) & =0 \\
Q_{1}(t, u) & =0
\end{aligned}
$$


or solutions of one of the equations

$$
\begin{aligned}
h_{1} & =0 \\
& \vdots \\
h_{r} & =0 .
\end{aligned}
$$

Substitute $d_{0}=2 m+2 n-1-d$ where $d=d_{1}+\cdots+d_{r}$. Since $P_{1}$ and $Q_{1}$ are relatively prime polynomials of degree $d_{0}$, they have at most $d_{0}{ }^{2}$ solutions and each $h_{i}$ has at most $d_{i}\left(d_{i}-1\right)$ isolated solutions. The number of all these solutions amount to $d_{0}^{2}+d_{1}\left(d_{1}-1\right)+\cdots+d_{r}\left(d_{r}-1\right)<\left(d_{0}+\cdots+d_{r}\right)^{2}<N+1$.

Thus, at least one of $\left(t_{i}, u_{i}\right)$ is the solution of $h_{j}$ which is not isolated. That is, for some $i, j$ we have $h_{j}\left(t_{i}, u_{i}\right)=0$ and one of the partial derivative, say $\frac{\partial h_{j}}{\partial u}\left(t_{i}, u_{i}\right)$, is not equal to zero. By the implicit function theorem, there is $\phi \in C^{1}$ such that $(t, \phi(t))$, for some neighborhood of $t_{i}$, is the solution of $h_{j}(t, u)$ and hence Equations (8) and (9). In addition, $L(t, u)=(\mathbf{r}(t)-\mathbf{s}(u)) \cdot(\mathbf{r}(t)-\mathbf{s}(u))$, under the constraints of (8) and (9), measures the diameter of the contact disks. If we substitute $u=\phi(t)$, then it is easy to see that $\frac{d L}{d t}(t, \phi(t))=0$ in a neighborhood of $t_{i}$, which means that $\Omega_{F}$ contains a parallel fundamental domain. By Theorem [11, the whole $\Omega_{F}$ must be parallel.

Let us now study the discrete critical horizontal sections in $\Omega_{F}$ as stated in Theorem 11. We first introduce an iterative procedure of securing regions that are free of any critical horizontal sections.

Let us continue to suppose $\kappa_{\mathbf{r}}>0$ and $\kappa_{\mathbf{s}}<0$ as in Case 3. Let $\alpha(t)$ be the angle of $\mathbf{r}^{\prime}(t)$ and $\beta(u)$ be that of $\mathbf{s}^{\prime}(u)$ as in the proof of Proposition 9. Assume also that total angle variations of $\mathbf{r}^{\prime}(t)$ and $\mathbf{s}^{\prime}(u)$ do not exceed $\pi$, i.e.,

$$
\begin{aligned}
\Delta \alpha & =\int_{0}^{1} \kappa_{\mathbf{r}}(t)\left|\mathbf{r}^{\prime}(t)\right| d t<\pi \\
\Delta \beta & =\int_{0}^{1} \kappa_{\mathbf{s}}(u)\left|\mathbf{s}^{\prime}(u)\right| d u>-\pi .
\end{aligned}
$$

The reason behind this assumption is that we do not want to deal with excessively bent fundamental domains. And this assumption can be easily satisfied by inserting some contact disks, if necessary, in the fundamental domain concerned. Now we are ready to start the procedure. Take $\mathbf{r}\left(t_{1}\right)$ for any $0<t_{1}<1$ and let $\mathbf{s}\left(u_{1}\right)$ be the corresponding contact point on $\mathbf{s}$. Let $\theta_{1}$ be the opening angle at $\mathbf{r}\left(t_{1}\right)$. Note that $\mathbf{r}^{\prime}(t)$ rotates positively and $\mathbf{s}^{\prime}(u)$ negatively as the corresponding parameters increase. Suppose first $\theta_{1}>\pi$. Since $\beta\left(u_{1}\right)=\alpha\left(t_{1}\right)+\theta_{1}$, the parameter $t_{2}$ such that $\alpha\left(t_{2}\right)+\pi=\beta\left(u_{1}\right)$, i.e., such that $\mathbf{r}^{\prime}\left(t_{2}\right)$ is parallel to $\mathbf{s}^{\prime}\left(u_{1}\right)$ satisfies $t_{2}>t_{1}$. Then we can claim that

Proposition 14. The fundamental domain defined by contact disks $\mathbf{r}\left(t_{1}\right)$ and $\mathbf{r}\left(t_{2}\right)$ has no critical horizontal sections.

Proof. Let $\mathbf{s}\left(u_{2}\right)$ be the corresponding contact point of $\mathbf{r}\left(t_{2}\right), \mathbf{p}$ be a medial axis point in the fundamental domain, and $\mathbf{r}(t)$ and $\mathbf{s}(u)$ be the contact points of $\mathbf{p}$. 


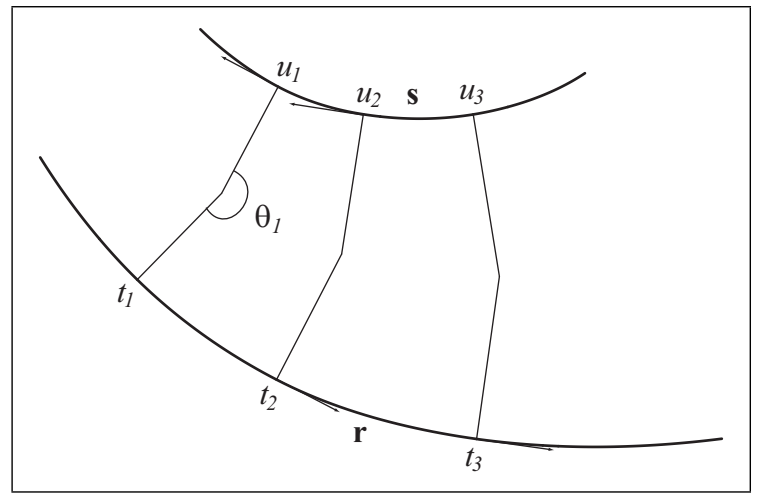

Figure 13: Monotone Region

To prove that $\mathbf{p}$ is not a critical horizontal section, we only need to show that the opening angle of $\mathbf{p}$, i.e., $\beta(u)-\alpha(t)$ never equals $2 k \pi+\pi$ for any integer $k$. Note that we have $t_{1}<t<t_{2}$ and $u_{1}>u>u_{2}$. Since $\beta\left(u_{1}\right)<\beta(u)<\beta\left(u_{2}\right)$ and $\alpha\left(t_{1}\right)<\alpha(t)<\alpha\left(t_{2}\right)$, we have $\beta\left(u_{1}\right)-\alpha\left(t_{2}\right)<\beta(u)-\alpha(t)<\beta\left(u_{2}\right)-\alpha\left(t_{1}\right)$. Now the result follows by the observation that $\beta\left(u_{1}\right)-\alpha\left(t_{2}\right)=\pi$ and $\beta\left(u_{2}\right)-\alpha\left(t_{1}\right)=$ $\beta\left(u_{2}\right)-\beta\left(u_{1}\right)+\theta_{1}<\pi+\theta_{1}<3 \pi$.

Let $\theta_{2}$ be the opening angle at $\mathbf{r}\left(t_{2}\right)$. Since $\theta_{2}>\pi$, by applying the above procedure recursively, we can obtain an increasing sequence $\left\{t_{k}\right\}$ such that the opening angle at $\mathbf{r}\left(t_{k}\right)$ is larger than $\pi$. In the same way, but in an opposite direction, we can also obtain a decreasing sequence $\left\{t_{k}^{\prime}\right\}$ such that $t_{1}^{\prime}=t_{1}$ and the opening angle at $\mathbf{r}\left(t_{k}^{\prime}\right)$ is larger than $\pi$. So, we can obtain two sequences $\left\{t_{k}\right\}$ and $\left\{t_{k}^{\prime}\right\}$ each of which is marching in each direction.

Now if $\theta_{1}<\pi$, the procedure follows similarly except that the opening angle is smaller than $\pi$. So, we can also obtain two sequences $\left\{t_{k}\right\}$ and $\left\{t_{k}^{\prime}\right\}$ each of which is marching in each direction.

Theorem 15. If the sequence $\left\{t_{k}\right\}$ ( or $\left\{t_{k}^{\prime}\right\}$ ) obtained from the above procedure converges to some $0 \leq \tau \leq 1$, then the medial axis transform point corresponding to $\mathbf{r}(\tau)$ is a critical horizontal section.

Proof. Since $\alpha\left(t_{k}\right)+\theta_{k}=\beta\left(u_{k}\right)$ and $\beta\left(u_{k}\right)-\alpha\left(t_{k+1}\right)=\pi$, we have $\alpha\left(t_{k+1}\right)-$ $\alpha\left(t_{k}\right)=\theta_{k}-\pi$. Furthermore, we have

$$
\alpha\left(t_{k+1}\right)-\alpha\left(t_{k}\right)=\int_{t_{k}}^{t_{k+1}} \kappa_{\mathbf{r}}(t)\left|\mathbf{r}^{\prime}(t)\right| d t
$$

Since $\theta(t)$ is continuous and $\theta\left(t_{k}\right)-\pi=\alpha\left(t_{k+1}\right)-\alpha\left(t_{k}\right)$, we must have $\theta(\tau)=$ $\pi$.

Given any fundamental domain $\Omega_{F}$ with $\kappa_{\mathbf{r}}>0$ and $\kappa_{\mathbf{S}}<0$, we first take the medial axis transform point corresponding to the boundary point $\mathbf{r}\left(\frac{1}{2}\right)$. From 
this medial axis transform point, we march (in both directions) to the nearest critical horizontal section in $\Omega_{F}$. If we are out of $\Omega_{F}$ during the march, then $\Omega_{F}$ has no critical horizontal section. Otherwise, if we come to a critical horizontal section $\mathbf{p}$, we keep the record of $\mathbf{p}$ and restart with the fundamental domain defined by $\mathbf{p}$ and one of the medial axis transform points $\mathbf{p}(0)$ and $\mathbf{p}(1)$ that is closer to $\mathbf{p}$. In this way, we can locate all critical horizontal sections in $\Omega_{F}$.

\section{Monotonic fundamental domain}

Following the procedures of the previous sections, we can decompose the original domain into many fundamental domains containing no critical horizontal sections, i.e., no local maximal or minimal radius component. Thus all of our fundamental domains have monotone increasing or decreasing radius function. We will call them monotonic fundamental domains.

Now we discuss how to utilize the monotonic fundamental domain $\Omega_{M F}$ to locate the offset curve segments in $\Omega_{M F}$. We can assume that a parameterization $(\mathbf{p}(v), r(v))$ of $\mathbf{M A T}\left(\Omega_{M F}\right)$, defined on the interval $[0,1]$, so chosen that $r(v)$ is monotone increasing. First of all, then, with the data of $r(0)$ and $r(1)$, we can answer the question whether or not there exists any self-intersection points of the $d$-offset curves. Recall that the self-intersection points of $d$-offset curves are the medial axis points with a $d$ radius value. We have also seen in Section 2 that $d$-offset curve is the envelope of $d$-cutoff of $\operatorname{MAT}\left(\Omega_{M F}\right)$.

Now, if $d<r(0)$, there exists no self-intersection point since $r(v)>d$ for all $0 \leq u \leq 1$. And the $d$-offset curves consist of a pair of curves $\mathbf{r}_{d}(t)$ and $\mathbf{s}_{d}(u)$ for $t, u \in[0,1]$. On the other hand, if $r(0) \leq d \leq r(1)$, then $r(v)=d$ for exactly one $v \in[0,1]$. (Unless $r(0)=r(1)$, i.e., $\Omega_{F}$ is a parallel fundamental domain. In this case, the $d$-offset curve is $\mathbf{M A}\left(\Omega_{M F}\right)$ itself.) Finally, if $r(1)<d$, then the $d$-cutoff of $\operatorname{MAT}\left(\Omega_{M F}\right)$ is an empty set, which means that there is no $d$-offset curve at all in $\Omega_{M} F$. We summarize these results as follows (see also Figures 14]15, and [16):

- $d<r(0)$ : Since $r(v)>d$ for all $v \in[0,1],(p(v), r(v)-d)$ is a parameterization of $\mathbf{M A T}\left(\Omega_{M F}\right)_{d}$ for $v \in[0,1]$, and there is no self-intersection points.

- $r(0) \leq d \leq r(1)$ : Since $r(v)$ is monotonic increasing, there exists unique $c \in[0,1]$ satisfying $r(c)=d$. Then $(p(v), r(v)-d)$ for $c \leq u \leq 1$ is a parameterization of $\operatorname{MAT}\left(\Omega_{M F}\right)_{d}$, and $\mathbf{p}(c)$ is the self-intersection point.

- $r(1)<d$ : Since $r(v)<d$ for all $v \in[0,1], \operatorname{MAT}\left(\Omega_{M F}\right)_{d}$ is empty, and so is the $d$-offset curve.

So, as we go through each monotonic fundamental domain $\Omega_{M F}$, one by one, of the original domain $\Omega$, we do nothing and just ignore it if $d>r(0)$ and $d>r(1)$. If $d<r(0)$ and $d<r(1)$, we compute $\mathbf{r}_{d}$ and $\mathbf{s}_{d}$ and record them as $d$-offset curve of $\Omega_{M F}$. Finally, if $r(0) \leq d \leq r(1)$ or $r(0) \geq d \geq r(1)$, we first check if $\Omega_{M F}$ is parallel. If so, $\mathbf{r}_{d}, \mathbf{s}_{d}$, and $\mathbf{M A}\left(\Omega_{M F}\right)$ are all the same 


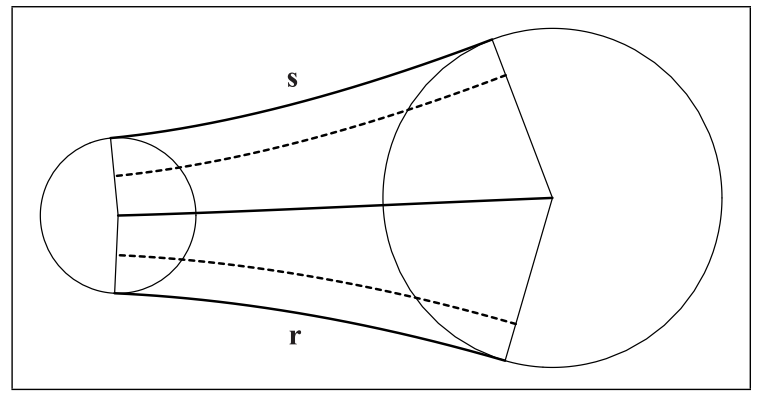

Figure 14: $d<r(0) \leq r(1)$

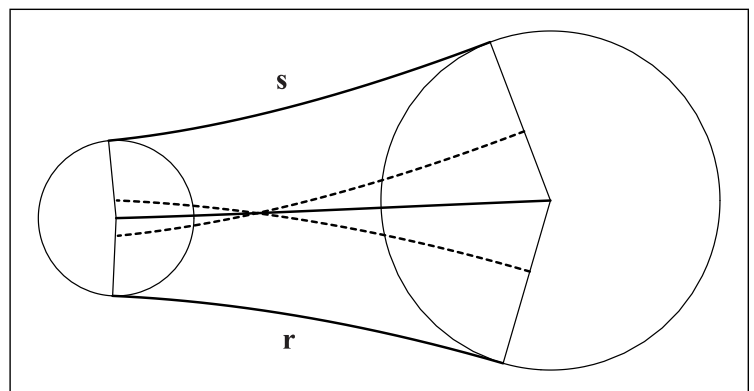

Figure 15: $r(0) \leq d \leq r(1)$

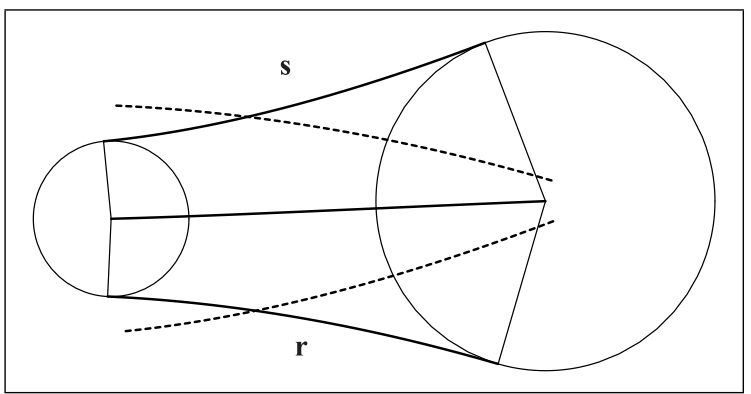

Figure 16: $r(0) \leq r(1)<d$

and each of them is the $d$-offset curve of $\Omega_{M F}$. If $\Omega_{M F}$ is not parallel, the unique self-intersection point can be located as follows. We can consider that $\operatorname{MAT}\left(\Omega_{M F}\right)$ is parameterized by $t$ or $u$, the parameters of the boundary curve $\mathbf{r}$ or $\mathbf{s}$, respectively. The fact that we can get the medial axis transform point $(\mathbf{p}(t), r(t))$ for any given boundary point $\mathbf{r}(t)$ for $t \in[0,1]$ amounts to that we can "evaluate" the $\mathbf{M A T}\left(\Omega_{M F}\right)$ as a function of $t$, especially the radius component $r(t)$. Recall that the self-intersection point is equivalent to solution of $r(t)=d$. Thus we can apply any numerical approximation scheme to solve this equation, for example the Newton-Raphson method. (Note that we can evaluate the 
derivative $r^{\prime}(t)$ by the Equation (4).) After we find the self-intersection point, or equivalently the contact disk with radius $d$, the monotonic fundamental domain $\Omega_{M F}$ is divided by the contact disk. The resulting two monotonic fundamental domains are just same as those considered above and we treat them accordingly. This procedure relies on numerical computation. Thus there may be numerical errors. However, the global, i.e., topological, nature remains unaltered.

\section{Illustrated example}

In Figure $17(\mathrm{a})$, a domain with a hole inside is decomposed into three fundamental domains. This process was addressed in 5. In Figure 17(b), we inserted three contact disks that correspond to the inflection points of the boundary curve. Now, the boundaries of the resulting fundamental domains have constant curvature signs. In Figure 17(c) all peaks and valleys are found, if any, in each fundamental domain. Now the resulting fundamental domains are monotonic fundamental domains. In Figure 17(d), the self-intersection points for a given value $d$ are found, if any, in each monotonic fundamental domain. Finally, in Figure 17(e), offset curves are computed in each valid fundamental domain.

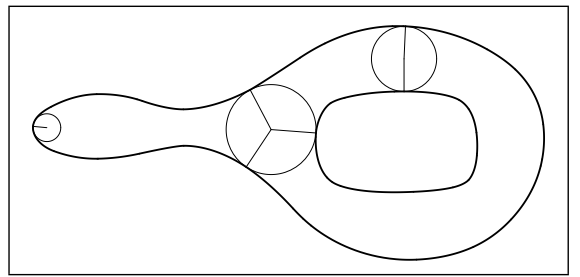

(a) Step 1 - Fundamental Domains

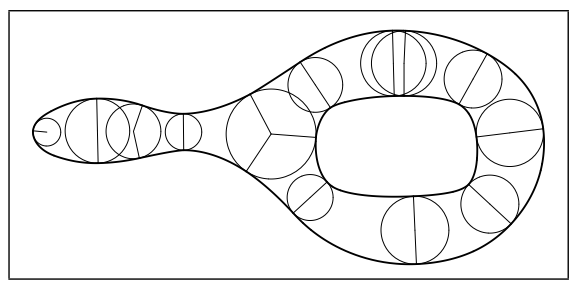

(c) Step 3 - Monotonic Fundamental Domains

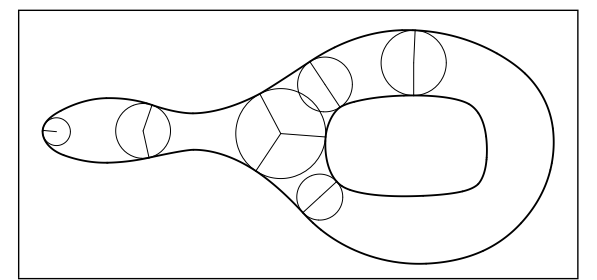

(b) Step 2 - Constant curvature sign

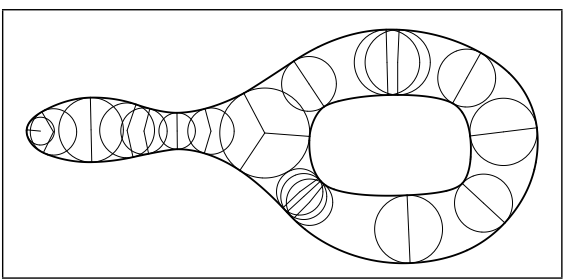

(d) Step 4 - Self-intersection points

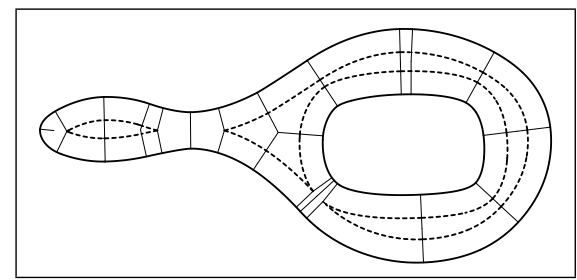

(e) Step 5 - Final offset curves 


\section{References}

[1] R. Ait-Haddou, L. Biard, and M. A. Slawinski. Minkowski isoperimetrichodograph curves. Computer Aided Geometric Design, 17(9):835-861, Oct 2000 .

[2] C.-S. Chiang, C. M. Hoffmann, and R. E. Lynch. How to compute offsets without self-intersection. Technical Report CSD-TR-91-072, Computer Sciences Department, Purdue University, October 1991.

[3] B. K. Choi and S. C. Park. A pair-wise offset algorithm for 2d pointsequence curve. Computer-Aided Design, 31(12):735-745, Oct 1999.

[4] H. I. Choi, S. W. Choi, and H. P. Moon. Mathematical theory of medial axis tranform. Pacific Journal of Mathematics, 181(1):57-88, 1997.

[5] H. I. Choi, S. W. Choi, H. P. Moon, and N.-S. Wee. New algorithm for medial axis transform of plane domain. Graphical Models and Image Processing, 59(6):463-483, November 1997.

[6] J. J. Chou and E. Cohen. Computing offsets and tool paths with Voronoi diagrams. Technical report, Department of Computer Science, University of Utah, Salt Lake City, UT 84112 USA, August 1990.

[7] S. H. F. Chuang and C. Z. Kao. One-sided arc approximation of b-spline curves for interference-free offsetting. Computer-Aided Design, 31(2):111118, Feb 1999.

[8] S. Coquillart. Computing offsets of B-spline curves. Computer-Aided Design, 19(6):305-309, Jul 1987.

[9] G. Elber, I.-K. Lee, and M.-S. Kim. Comparing offset curve approximation methods. IEEE Computer Graphics and Applications, 17(3):62-71, MayJun 1997.

[10] R. T. Farouki. Conic approximation of conic offsets. Journal of Symbolic Computation, 23:301-313, 1997.

[11] R. T. Farouki and C. A. Neff. Algebraic properties of plane offset curves. Computer Aided Geometric Design, 7:101-127, 1990.

[12] R. T. Farouki and C. A. Neff. Analytic properties of plane offset curves. Computer Aided Geometric Design, 7:83-99, 1990.

[13] R. T. Farouki and R. Ramamurthy. Specified-precision computation of curve/curve bisector. Technical Report UM-MEAM-96-10, Dept. of Mechanical Engineering and Applied Mechanics, University of Michigan, Ann Arbor, MI 48109.

[14] R. T. Farouki and S. T. Pythagorean hodographs. IBM Journal of Research and Development, 34:736-752, 1990. 
[15] H.-Y. Feng and H. Li. Constant scallop-height tool path generation for three-axis sculptured surface machining. Computer-Aided Design, 34(9):647-654, Aug 2002.

[16] G. Glaeser, J. Wallner, and H. Pottmann. Collision-free 3-axis milling and selection of cutting tools. Computer-Aided Design, 31(3):225-232, Mar 1999.

[17] M. Held. On the Computational Geometry of Pocket Machining. Lecture Notes in Computer Science 500. Springer-Verlag, 1991.

[18] M. Held, G. Lukács, and L. Andor. Pocket machining based on coutourparallel tool paths generated by means of proximity maps. Computer-Aided Design, 26(3):189-203, March 1994.

[19] V. D. Holla, K. G. Shastry, and B. G. Prakash. Offset of curves on tessellated surfaces. Computer-Aided Design, 35(12):1099-1108, Oct 2003.

[20] H.-W. L. Hon-Yuen Tam and H. Xu. A geometric approach to the offsetting of profiles on three-dimensional surfaces. Computer-Aided Design, 36:887902, 2004.

[21] J. Hoschek. Offset curves in the plane. Computer-Aided Design, 17(2):7782, March 1985.

[22] J. Hoschek. Spline approximation of offset curves. Computer Aided Geometric Design, 5:33-40, 1988.

[23] K. C. Hui. Free-form design using axial curve-pairs. Computer-Aided Design, 34(8):583-595, July 2002.

[24] C.-S. Jun, D.-S. Kim, and S. Park. A new curve-based approach to polyhedral machining. Computer-Aided Design, 34(5):379-389, Api 2002.

[25] B. Jüttler and C. Mäurer. Cubic pythagorean hodograph spline curves and applications to sweep surface modeling. Computer-Aided Design, 31(1):7383, Jan 1999.

[26] R. Klass. An offset spline approximation for plane cubic splines. ComputerAided Design, 15(5):297-299, September 1983.

[27] C. Lartigue, F. Thiebaut, and T. Maekawa. Shapes with offsets of nearly constant surface area. Computer-Aided Design, 31(4, 1):287-296, Apr 1999.

[28] C. Lartigue, F. Thiebaut, and T. Maekawa. Cnc tool path in terms of b-spline curves. Computer-Aided Design, 33(4, 2):307-319, Apr 2001.

[29] E. Lee. Contour offset approach to spiral toolpath generation with constant scallop height. Computer-Aided Design, 35(6):511-518, May 2003. 
[30] I.-K. Lee, M.-S. Kim, and G. Elber. Planar curve offset based on circle approximation. Computer-Aided Design, 28(8):617-630, Aug 1996.

[31] D. S. Meek and D. J. Walton. Offset curves of clothoidal splines. ComputerAided Design, 22(4):199-201, May 1990.

[32] S. C. Park and B. K. Choi. Uncut free pocketing tool-paths generation using pair-wise offset algorithm. Computer-Aided Design, 33(10):739-748, Sep 2001.

[33] S. C. Park and Y. C. Chung. Offset tool-path linking for pocket machining. Computer-Aided Design, 34(4):299-308, Api 2002.

[34] S. C. Park, Y. C. Chung, and B. K. Choi. Contour-parallel offset machining without tool-retractions. Computer-Aided Design, 35(9):841-849, Aug 2003.

[35] H. Persson. NC machining of arbitrarily shaped pockets. Computer-Aided Design, 10(3):169-174, May 1978.

[36] B. Pham. Offset approximation of uniform B-splines. Computer-Aided Design, 20(8):471-474, Oct 1988.

[37] B. Pham. Offset curves and surfaces: A brief survey. Computer-Aided Design, 24(4):223-229, Apr 1992.

[38] L. A. Piegl and W. Tiller. Computing offsets of NURBS curves and surfaces1. Computer-Aided Design, 31(2):147-156, Feb 1999.

[39] H. Pottmann. Curve design with rational Pythagorean-hodograph curves. Advances in Computational Mathematics, 3:147-170, 1995.

[40] H. Pottmann. Rational curves and surfaces with rational offsets. Computer Aided Geometric Design, 12(2):175-192, Mar 1995.

[41] G. V. V. Ravi Kumar, K. G. Shastry, and B. G. Prakash. Computing non-self-intersecting offsets of NURBS surfaces. Computer-Aided Design, 34(3):209-228, Mar 2002.

[42] W. Tiller and E. G. Hanson. Offsets of two-dimensional profiles. IEEE Computer Graphics and Applications, 4:36-46, 1984.

[43] L. Z. Wang, K. T. Miura, E. Nakamae, T. Yamamoto, and T. J. Wang. An approximation approach of the clothoid curve defined in the interval $[0, / 2]$ and its offset by free-form curves. Computer-Aided Design, 33(14):10491058, Dec 2001. 